\title{
Cosmology for Particle Physicists
}

\author{
U. A. Yajnik \\ Indian Institute of Technology, Bombay, Mumbai
}




\section{Contents}

1 Introduction 4

1.1 General Theory of Relativity : A Recap . . . . . . . . . . . . . . 4

1.1.1 True or False . . . . . . . . . . . . . . . . . . 5

1.2 The Standard Model of Cosmology . . . . . . . . . . . . . . . . . . . . . . . . . . 5

1.3 The Standard Model of Particle Physics . . . . . . . . . . . . . . . . . . . . 7

2 Friedmann-Robertson-Walker Metrics 7

2.1 Cosmological redshift . . . . . . . . . . . . . . . . . . . . . . . . . . . . . . . . . .

2.2 Luminosity Distance . . . . . . . . . . . . . . . . . . . . 9

3 The Three Laws of Cosmology $\quad \mathbf{1 0}$

3.1 Example : Friedman Universe ...................... 11

3.2 Parameters of the Universe . . . . . . . . . . . . . . . . . . 12

4 The Big Bang Universe $\quad 012$

4.1 Thermodynamic Relations . . . . . . . . . . . . . . . . . . . 13

4.2 Isentropic Expansion . . . . . . . . . . . . . . . . . . . . . . . . . . . . . . . . . . . . . .

4.3 Temperature Thresholds . . . . . . . . . . . . . . . . . . . 14

4.4 Photon Decoupling and Recombination . . . . . . . . . . . . . . . . . . 14

4.4.1 Saha Equation for Ionization Fraction . . . . . . . . . . . . . 15

5 Phase Transitions with Quantum Fields in the Early Universe 16

5.1 Legendre Transform . . . . . . . . . . . . . . . . . . . . . . . . . . . . . . .

5.2 Effective action and effective potential . . . . . . . . . . . . . . . 17

5.3 Computing $V_{e f f} \ldots \ldots \ldots \ldots \ldots \ldots \ldots \ldots$

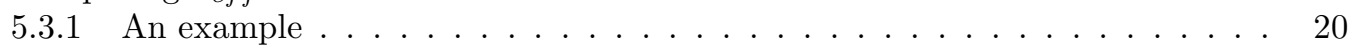

5.4 Temperature corrections to 1 -loop . . . . . . . . . . . . . . . . . 21

6 First order Phase transition $\quad \mathbf{2 3}$

6.1 Tunneling ......................... 24

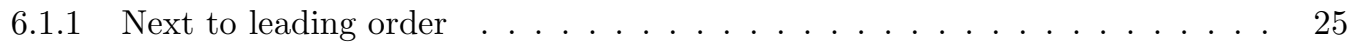

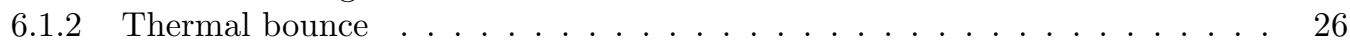

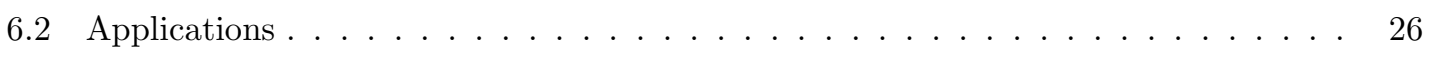

7 Inflationary Universe $\quad \mathbf{2 7}$

7.1 Fine tuned initial conditions . . . . . . . . . . . . . . . . . . . . . . . . . . . .

7.2 Horizon problem . . . . . . . . . . . . . . . . . . . . . . . . . . . . . . . . . . . . . .

7.3 Oldness-Flatness problem . . . . . . . . . . . . . . . . . . . 28

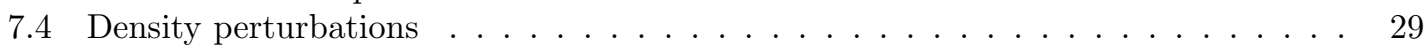

7.5 Inflation . . . . . . . . . . . . . . . . . . . . . 29

7.6 Resolution of Problems . . . . . . . . . . . . . . . . . . . . . . . . . . . . . . . . . . . . . .

7.6.1 Horizon and flatness problems . . . . . . . . . . . . . . . . . . . . . . . . .

7.6 .2 Avoidance of unwanted relics . . . . . . . . . . . . . . . . . . . . . . . . . . . . . .

7.6.3 Resolution for density perturbations . . . . . . . . . . . . . . . 32

7.7 Inflaton Dynamics . . . . . . . . . . . . . . . . . . . . . . . . . . . . . . 33

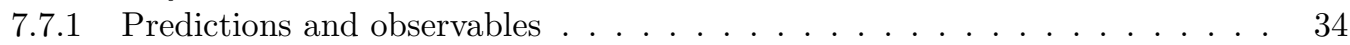

8 Density perturbations and galaxy formation $\quad 36$

8.1 Jeans Analysis for Adiabatic Perturbations . . . . . . . . . . . . . . . . . . . . . . . . . 36

8.2 Jeans Analysis in Expanding Universe . . . . . . . . . . . . . . . . . . . . . . . . . . . . 37

8.2.1 Fate of the super-horizon modes . . . . . . . . . . . . . . . . . . . . . . . . .

8.2.2 Connection to density and temperature perturbations . . . . . . . . . . 39

8.3 Density Fluctuations from Inflation . . . . . . . . . . . . . . . . . . . 40 
9 Relics of the Big Bang 42

9.1 Boltzmann Equations . . . . . . . . . . . . . . . . . . 42

9.1.1 Freeze out and subsequent evolution . . . . . . . . . . . . . . 44

9.2 Dark Matter . . . . . . . . . . . . . . . . . . . . 45

9.2 .1 Hot Relics . . . . . . . . . . . . . . . . . . . . . . 47

9.2 .2 Cold Relics . . . . . . . . . . . . . . . . . . . 47

10 Missing Dark Energy 48

11 Baryon asymmetry of the Universe 48

11.1 Genesis of the idea of Baryogenesis . . . . . . . . . . . . . . 48

11.2 Sakharov criteria . . . . . . . . . . . . . . . . . . . . . 49

11.3 Anomalous violation of $B+L$ number $\ldots \ldots \ldots \ldots \ldots \ldots \ldots$

11.4 Electroweak baryogenesis . . . . . . . . . . . . . . . . . 50

11.5 Baryogenesis from Leptogenesis . . . . . . . . . . . . . . . . . . 51

12 Appendix 53 


\section{Introduction}

Over the past two decades Cosmology has increasingly become a precision science. That the Universe is expanding was an astonishing discovery. Now we know its details to unprecedented precision. An expanding Universe also implied an extremely compact state in the past, and therefore very high temperature. The Particle Physics forces which can now be explored only in accelerator laboratories were in free play in the remote past. Thus the observation of the oldest remnants in the Universe amounts to looking at the results of a Particle Physics experiment under natural conditions.

In these notes we present a selection of topics, each section approximately amounting to one lecture. We begin with a brief recapitulation of General Relativity, and the Standard Model of Cosmology. The study of Cosmology requires General Relativity to be applied only under a highly symmetric situation and therefore it is possible to recast the essentials as Three Laws of Cosmology. The study of very early Universe brings us squarely into the domain of Quantized Field Theory at given temperature. Intermediate metastable phases through which the Universe passed require an understanding of the effective potential of the field theory in a thermal equilibrium. This formalism is developed in some detail.

The remainder of the notes discuss important signatures of the remote past. These include : (i) inflation, (ii) density perturbations leading to galaxy formation, (iii) study of hot and cold relics decoupled from the remaining constituents, some of which can be candidates for Dark Matter, (iv) finally the baryon asymmetry of the Universe. As we shall see each of these has a strong bearing on Particle Physics and is being subjected to ever more precise observations.

\subsection{General Theory of Relativity : A Recap}

Special Theory of Relativity captures the kinematics of space-time observations. On the other hand, General Theory of Relativity is a dynamical theory, which extends the Newtonian law of gravity to make it consistent with Special Relativity. In this sense it is not a "generalization" of Relativity but rather, a theory of Gravity on par with Maxwell's theory of Electromagnetism. It is nevertheless a very special kind of theory because of the Principle of Equivalence. The equivalence of gravitational and inertial masses ensures that in a given gravitational field, all test particles would follow a trajectory decided only by their initial velocities, regardless of their mass. This makes it possible to think of the resulting trajectory as a property of the spacetime itself. This was the motivation for introducing methods of Differential Geometry, the mathematics of curved spaces for the description of Gravity. Due to this "grand unification" of space and time into a dynamically varying set we shall use the convention of writing the word spacetime without hyphenation as adopted in ref. [1].

Throughout these notes we use the convention $\hbar=c=1$ and the sign convention $d s^{2}=$ $d t^{2}-|d \mathbf{x}|^{2}$ for the spacetime interval in Special Relativity. The principle of General Covariance states that a given gravitational field is described by a metric tensor $g_{\mu \nu}$ a function of spacetime variables collectively written as $x^{\mu} \equiv \mathbf{x}, t$. Gravity modifies the spacetime interval to the general quadratic form $g_{\mu \nu} d x^{\mu} d x^{\nu}$, where the summation convention on same indices is assumed. The trajectories of test particles are simply the shortest possible paths in this spacetime, determined by the metric tensor through the geodesic equation

$$
\frac{d^{2} x^{\mu}}{d \tau^{2}}+\Gamma_{\nu \rho}^{\mu} \frac{d x^{\nu}}{d \tau} \frac{d x^{\rho}}{d \tau}=0
$$

where the Christoffel symbols $\Gamma_{\nu \rho}^{\mu}$ are given by

$$
\Gamma_{\nu \rho}^{\mu}=\frac{1}{2} g^{\mu \lambda}\left(\frac{\partial g_{\nu \lambda}}{\partial x^{\rho}}-\frac{\partial g_{\nu \rho}}{\partial x^{\lambda}}+\frac{\partial g_{\rho \lambda}}{\partial x^{\nu}}\right)
$$

These symbols are not tensors but determine the covariant derivative much the same way that the electromagnetic potentials which are themselves not gauge invariant determine the minimal coupling of charged particles to electromagnetic fields. 
The equations which determine the gravitational field, i.e., the tensor $g_{\mu \nu}$ itself are the Einstein Equations,

$$
G_{\mu \nu}-\Lambda g_{\mu \nu} \equiv R_{\mu \nu}-\frac{1}{2} g_{\mu \nu} R-\Lambda g_{\mu \nu}=8 \pi G T_{\mu \nu}
$$

where $T_{\mu \nu}$ is the energy momentum tensor and the Ricci tensor $R_{\mu \nu}$ and the scalar curvature $R$ are the contracted forms of the fourth rank tensor the Riemann curvature, given by

$$
\begin{aligned}
R_{\nu \alpha \beta}^{\mu} & =\partial_{\alpha} \Gamma_{\nu \beta}^{\mu}-\partial_{\beta} \Gamma_{\nu \alpha}^{\mu}+\Gamma_{\sigma \alpha}^{\mu} \Gamma_{\nu \beta}^{\sigma}-\Gamma_{\sigma \beta}^{\mu} \Gamma_{\nu \alpha}^{\sigma} \\
R_{\mu \nu} & =R_{\mu \lambda \nu}^{\lambda} \\
R & =g^{\mu \nu} R_{\mu \nu}
\end{aligned}
$$

The tensor $G_{\mu \nu}$ is called the Einstein tensor and has the elegant property that its covariant derivative vanishes. The last term on the left hand side is called the Cosmological term since its effects are not seen on any small scale, even galactic scales. It can be consistently introduced into the equations provided $\Lambda$ is a constant. Since the covariant derivative $D_{\rho} g_{\mu \nu}=0$, the covariant derivative of this term also vanishes. This fact is matched on the right hand side of Einstein's equation by the vanishing of the covariant derivative of the energy-momentum tensor. The vanishing of the Einstein tensor follows from the Bianchi identities in Differential Geometry. The geometric significance of the identities is that given a spacetime domain, they express the statement "the boundary of a boundary is zero". We leave it to the reader to pursue ref [1] to understand its details. Thus a geometric principle implies the covariant conservation of energymomentum tensor, a physical quantity. But it has to be noted that covariant conservation does not imply a conserved charge the way it happens in flat spacetime with divergence of a four-vector. But if there are 3-dimensional regions on whose 2-dimensional boundaries gravity is very weak, it does imply conservation of total mass-energy in the given volume.

There are quite a few subtleties concerning the implications of General Relativity and the conditions under which it supercedes Newtonian gravity. We present here a few "True or False" statements for the reader to think over and discuss with peers or teachers. Starting points to answers are given in Appendix, sec. 12.

\subsubsection{True or False}

1. Curved spacetime is a necessity of GR due to the underlying Special Relativity principle.

2. The invariance of the equations of physics under arbitrary reparameterisation of spacetime is the essential new content of GR.

3. The notion of energy density becomes meaningless in GR

4. The notion of total energy becomes meaningless in GR

5. Points where some of the metric coefficients vanish are unphysical

6. Points where some of the metric coefficients diverge are unphysical

7. Points where any components of curvature tensor diverge are unphysical

8. Newtonian gravity is insufficient to describe an expanding Universe and GR is required.

\subsection{The Standard Model of Cosmology}

Here we summarise the broadest features of our current understanding of the Universe based on a vast variety of data and modeling. We summarise size, age and contents of the Universe as follows 
- There is a strong indication that the Universe is homogeneous and isotropic if we probe it at sufficiently large scales, such as the scale of clusters of galaxies. The typical scale size is 10 Megaparsec (Mpc) and larger. At the scale of several tens of Mpc the distribution of galaxies is homogeneous.

It is at present not known whether the Universe is finite in size or infinite. A finite Universe would be curved as a three dimensional manifold. An infinite universe could also show curvature. At present we do not see any signs of such curvature.

- Secondly we believe that the Universe has been expanding monotonically for a finite time in the past. This gives a finite age of about 13.7 billion years to our Universe.

What was "before" this time is not possible to understand within the framework of classical General Relativity. But Newton's gravitational constant suggests a fundamental mass scale, called the Planck scale $M_{P l}=G^{-1 / 2} \approx 1.2 \times 10^{19} \mathrm{GeV}$ and corresponding extremely small time scale, $10^{-44} \mathrm{sec}$. We expect that Quantum theory of Gravity should take over at that scale. Unfortunately that theory has not yet been worked out due to insufficient data.

- Finally it has been possible to map the current contents of the Universe to a reasonable accuracy. More about them below. The contents of the Universe can be divided into three types,

1. Radiation and matter as known in Particle Physics

2. Dark Matter, one or more species of particles, at least one of which is necessarily nonrelativistic and contributing significantly to the total energy density of the Universe today. These particles have no Standard Model interactions.

3. Dark Energy, the largest contributor to the energy density balance of the present Universe, a form of energy which does not seem to fit any known conventional fields or particles. It could be the discovery of a non-zero cosmological constant. But if more precise observations show its contribution to be changing with time, it can be modelled as a relativistic continuum which possesses negative pressure.

Of the contents of type 1 , there are approximately 400 photons per cc and $10^{-7}$ protons per cc on the average. Compared to Avogadro number available on earth, this is a very sparse Universe. Of these the major contributor to energy density is baryonic matter. This constitutes stars, galaxies and large Hydrogen clouds out of which more galaxies and stars are continuously in formation. The other component is the Cosmic Microwave Background Radiation (CMBR), the gas of photons left over after neutral Hydrogen first formed. Its contribution to total energy density is relatively insignificant. But its precision study by experiments such as the Wilkinson Microwave Anisotropy Probe ( WMAP) is providing us with a very detailed information of how these photons have been created and what are the ingredients they have encountered on their way from their origin to our probes.

There probably are other exotic forms of energy-matter not yet discovered. Two principle candidates are topological defects such as cosmic strings, and the axion. We shall not be able to discuss these here. Axions are almost a part of Standard Model though very weakly interacting, and could potentially be Dark Matter candidates.

Aside from these current parameters of the Universe there is a reason to believe that the Universe passed through one or more critical phases before arriving at the vast size and great age it presently has. This critical phase in its development is called Inflation. It is expected to have occurred in remote past at extremely high energies, perhaps in the Planck era itself. What is interesting is that the fluctuations in energy density which finally became galaxies could have originated as quantum effects during that era. Thus we would be staring at the results of quantum physics in the very early Universe whenever we see galaxies in the sky.

A quantitative summary of present observables of the Universe is given at the end of sec. 3 . 


\subsection{The Standard Model of Particle Physics}

We assume that the reader is familiar with the Standard Model of Particle Physics. Appendix B of Kolb and Turner contains a review. Cosmology has a dual role to play in our understanding of the fundamental forces. It is presenting us with the need to make further extensions of the Standard Model and is also providing evidence to complete our picture of Particle Physics. For example whether Dark Matter emerges from an extension of the Standard Model is a challenge to model building. On the other hand axions expected from QCD may perhaps get verified in Astroparticle physics experiments and may have played a significant role in the history of the Cosmos.

Study of Cosmology also sharpens some of the long recognized problems and provides fresh perspectives and fresh challenges. Symmetry breaking by Higgs mechanism in Standard Model (and its extensions the Grand Unified models) causes hierarchy problem. But it also implies a cosmological constant far larger than observed. We hope that the two problems have a common solution. Despite the conceptual problems with the QFT of scalar fields, inflation is best modeled by a scalar field. Similarly, consider Dark Energy which is almost like a cosmological constant of a much smaller value than Particle Physics scales. This has finally been confirmed over the past decade. Again many models seem to rely on unusual dynamics of a scalar field to explain this phenomena. We hope that supersymmetry or superstring theory will provide natural candidates for such scalar fields without the attendant QFT problems.

\section{Friedmann-Robertson-Walker Metrics}

Cosmology began to emerge as a science after the construction of reflection telescopes of 100 to 200 inch diameter in the USA at the turn of 1900. When Doppler shifts of Hydrogen lines of about twenty nearby galaxies could be measured it was observed that they were almost all redshifts. Edwin Hubble proposed a linear law relating redshift and distance. Then the data could be understood as a universal expansion. Over the last 75 years this fact has been further sharpened, with more than 10 million galaxies observed and cataloged.

It is reasonable to believe that we are not in a particularly special galaxy. So it is reasonable to assume that the expansion is uniform, i.e. observer on any other galaxy would also see all galaxies receding from him or her at the same rate. This reasoning allows us to construct a simple solution to Einstein's equations. We assume that the Universe corresponds to a solution in which aside from the overall expansion as a function of time, the spacetime is homogeneous and isotropic. This would of course be true only for the class of observers who are drifting along with the galaxies in a systematic expansion. ( An intergalactic spaceship moving at a high speed would see the galactic distribution quite differently). We characterize this coordinate system as one in which (1) there is a preferred time coordinate 1 such that at a given instant of this time, (2) the distribution of galaxies is homogeneous and isotropic.

It should be noted that this is a statement about the symmetry of the sought after solution of the Einstein equations. The symmetries restrict the boundary conditions under which the equations are to be solved and in this way condition the answer. In older literature the existence of such symmetries of the observed Universe was referred to as Cosmological Principle, asserted in the absence of any substantial data. Over the years with accumulation of data we realize that it is not a principle of physics, but a useful model suggested by observations, similar to the assumption that the earth is a spheroid.

The assumptions of homogeneity and isotropy made above are very strong and we have a very simple class of metric tensors left as possible solutions. They can be characterized by spacetime interval of the form

$$
d s^{2}=d t^{2}-R^{2}(t)\left\{\frac{d r^{2}}{1-k r^{2}}+r^{2} d \theta^{2}+r^{2} \sin ^{2} \theta d \phi^{2}\right\}
$$

\footnotetext{
${ }^{1}$ This time coordinate is not unique. We can define a new time coordinate $\tilde{t}(t)$ as an arbitrary smooth function of the old time coordinate $t$. What is unique is that a time axis is singled out from the spacetime continuum. What units, (including time dependent ones), we use to measure the time is arbitrary.
} 
The only dynamical degree of freedom left is the scale factor $R(t)$. Further, there are three possibilities distinguished by whether the spacelike hypersurface at a given time is flat and infinite ( Newtonian idea), or compact like a ball of dimension 3 with a given curvature, (generalization of the 2 dimensional shell of a sphere in usual 3 dimensional Euclidean space), or unbounded and with constant negative curvature, a possibility more difficult to visualize. These three possibilities correspond to the parameter $k=0$, or $k=+1$, or $k=-1$. The cases $k=-1$ and $k=1$ also have representations which make their geometry more explicit

$$
d s^{2}= \begin{cases}d t^{2}-R^{2}(t)\left\{d \chi^{2}+\sin ^{2} \chi\left(d \theta^{2}+\sin ^{2} \theta d \phi^{2}\right)\right\} & k=1 \\ d t^{2}-R^{2}(t)\left\{d \chi^{2}+\sinh ^{2} \chi\left(d \theta^{2}+\sin ^{2} \theta d \phi^{2}\right)\right\} & k=-1\end{cases}
$$

The time coordinate $t$ we have used above is a particular choice and is called comoving time. An alternative time coordinate $\eta$ is given by

$$
\begin{gathered}
d \eta=\frac{d t}{R(t)} \\
d s^{2}=R^{2}(\eta)\left\{d \eta^{2}-\frac{d r^{2}}{1-k r^{2}}-d r^{2}-r^{2} \sin ^{2} \theta d \phi^{2}\right\}
\end{gathered}
$$

Its advantage is that for $k=0$ it makes the metric conformally equivalent to flat (Minkowski ) space.

\subsection{Cosmological redshift}

In this and the next subsection we identify the precise definitions of redshift and cosmological distances to understand Hubble Law in its general form.

The observed redshift of light is similar to Doppler shift, but we would like to think that it arises because spacetime itself is changing and not due to relative motion in a conventional sense. In other words, in cosmological context the redshift should be understood as arising from the fact that time elapses at a different rate at the epoch of emission $t_{1}$ from that at the epoch $t_{0}$ of observation. Since light follows a geodesic obeying $d s^{2}=0$, it is possible for us to define the quantity $f\left(r_{1}\right)$ which is a dimensionless measure of the separation between emission point $r=0$ and the observation point $r=r_{1}$

$$
f\left(r_{1}\right) \equiv \int_{0}^{r_{1}} \frac{d r}{\left(1-k r^{2}\right)^{1 / 2}}=\int_{t_{1}}^{t_{0}} \frac{d t}{R(t)}
$$

where the second equality uses $d s^{2}=0$. Now the same $f\left(r_{1}\right)$ is valid for a light signal emitted a little later at $t=t_{1}+\delta t_{1}$ and received at a corresponding later time $t_{0}+\delta t_{0}$.

$$
f\left(r_{1}\right)=\int_{t_{1}+\delta t_{1}}^{t_{0}+\delta t_{0}} \frac{d t}{R(t)}
$$

Equivalently,

$$
\begin{aligned}
\int_{t_{1}}^{t_{1}+\delta t_{1}} \frac{d t}{R} & =\int_{t_{0}}^{t_{0}+\delta t_{0}} \frac{d t}{R(t)} \\
\frac{\delta t_{1}}{R\left(t_{1}\right)} & =\frac{\delta t_{0}}{R\left(t_{0}\right)}
\end{aligned}
$$


or

$$
\frac{\nu_{0}}{\nu_{1}}=\frac{\delta t_{1}}{\delta t_{0}}=\frac{\lambda_{1}}{\lambda_{0}}=\frac{R\left(t_{1}\right)}{R\left(t_{0}\right)}
$$

It is convenient to define the redshift $z$, originally so defined because it would always be small, as given by

$$
1+z \equiv \frac{\lambda_{0}}{\lambda_{1}}=\frac{R\left(t_{0}\right)}{R\left(t_{1}\right)}
$$

\subsection{Luminosity Distance}

Defining a measure of spacelike distances is tricky in Cosmology because physical separations between comoving objects are not static and therefore lack an operational meaning. Since distances are measured effectively by observing light received, we define luminosity distance $d_{L}$ by

$$
\begin{aligned}
d_{L}^{2}=\frac{L}{4 \pi F} \rightarrow \text { absolute luminosity } \\
\rightarrow \text { observed flux }
\end{aligned}
$$

If the metric were frozen to its value at time $t_{0}$ this would have been the same as in flat space, $R\left(t_{0}\right)^{2} r_{1}^{2}$ with $r_{1}$ the coordinate distance travelled by light.Due to expansion effects, we need additional factors of $1+z$, once for reduction in energy due to redshift and once due to delay in the observation of the signal

$$
d_{L}^{2}=R^{2}\left(t_{0}\right) r_{1}^{2}(1+z)^{2}
$$

We now introduce measures $H_{0}$ for the first derivative, representing the Hubble parameter and a dimesionless measure $q_{0}$ for the second derivative, traditionally deceleration, by expanding the scale factor as

$$
\begin{gathered}
\frac{R(t)}{R\left(t_{0}\right)}=1+H_{0}\left(t-t_{0}\right)-\frac{1}{2} q_{0} H_{0}^{2}\left(t-t_{0}\right)^{2}+\ldots \\
H_{0} \equiv \frac{\dot{R}\left(t_{0}\right)}{R\left(t_{0}\right)} \quad q_{0} \equiv-\frac{\ddot{R}\left(t_{0}\right)}{\dot{R}^{2}\left(t_{0}\right)} R\left(t_{0}\right)=\frac{-\ddot{R}}{R H_{0}^{2}} \\
\therefore z=H_{0}\left(t_{0}-t\right)+\left(1+\frac{q_{0}}{2}\right) H_{0}^{2}\left(t_{0}-t\right)^{2}+\ldots
\end{gathered}
$$

so that

$$
\left(t_{0}-t\right)=H_{0}^{-1}\left(Z-\left(1+\frac{q_{0}}{2}\right) Z^{2}+\ldots\right)
$$

We now use the quantity $f(r)$ introduced in the discussion of redshift of light, which for the three different geometries works out to be

$$
\begin{gathered}
f\left(r_{1}\right)=\left\{\begin{array}{c}
\sin ^{-1} r_{1}=r_{1}+\frac{|r|^{3}}{6} \\
r_{1} \\
\sinh ^{-1}=-\frac{1}{6}
\end{array}\right. \\
\therefore r_{1}=\frac{1}{R\left(t_{0}\right)}\left[\left(t_{0}-t_{1}\right)+\frac{1}{2} H_{0}\left(t_{0}-t_{1}\right)^{2}+\ldots\right]
\end{gathered}
$$

Substitute $\left(t_{0}-t_{1}\right)$

$$
\begin{aligned}
r_{1} & =\frac{1}{R\left(t_{0}\right) H_{0}}\left[z-\frac{1}{2}\left(1+q_{0}\right) z^{2}+\ldots\right] \\
H_{0} d_{L} & =z+\frac{1}{2}\left(1-q_{0}\right) z^{2}+\ldots
\end{aligned}
$$


The last relation expresses the connection between observed luminosity of distance $d_{L}$ of galaxies and their redshift $z$, incorporating the curvature effects arising from the expansion of the Universe. Extensive data on $d_{L}$ and $z$ gathered from galaxy surveys such as 2-degree Field Galactic Redshift Survey ( $2 \mathrm{dF}$ GRS) and 6dF GRS can be fitted with this equation to determine cosmological parameters $H_{0}$ and $q_{0}$.

\section{The Three Laws of Cosmology}

It is possible to discuss the cosmological solution without recourse to the full Einstein equations. After all a comprehensive framework like electromagnetism was discovered only as a synthesis of a variety of laws applicable to specific situations. Specifically, Coulomb's law is most useful for static pointlike charges. Similarly, given that the Universe has a highly symmetric distribution of matter, allows us to express the physics as three laws applicable to Cosmology, these being

1. Evolution of the scale factor : The evolution of $R(t)$ introduced above is governed by

$$
\left(\frac{\dot{R}}{R}\right)^{2}+\frac{k}{R^{2}}-\frac{\Lambda}{3}=\frac{8 \pi}{3} G \rho
$$

2. Generalized thermodynamic relation : The energy-matter source of the gravitational field obeys generalization of the first law of thermodynamics $d U=-p d V$,

$$
\frac{d}{d t}\left(\rho R^{3}\right)+p \frac{d}{d t}\left(R^{3}\right)=0
$$

To put this to use we need the equation of state $p=p(\rho)$. For most purposes it boils down to a relation

$$
p=w \rho
$$

with $w$ a constant.

3. Entropy is conserved : (except at critical thresholds)

$$
\frac{d}{d t}(S)=\frac{d}{d t}\left(\frac{R^{3}}{T}(\rho+p)\right)=0
$$

The law 2 can be used to solve for $\rho$ as a function of $R$ with $w$ a given parameter. Then this $\rho$ can be substituted in law 1 to solve for $R(t)$ the cosmological scale factor.

As for Law 3, in the cosmological context we speak of entropy density $s$. Thus the above law applies to the combination $s R^{3}$. Further, non-relativistic matter does not contribute significantly to entropy while for radiation $s \propto T^{3}$. Hence we get the rule of thumb

$$
S=s R^{3} \propto T(t)^{3} R(t)^{3}=\text { constant }
$$

Thus Law 3 provides the relation of the temperature and the scale factor. However, critical threshold events are expected to have occured in the early Universe, due to processes going out of equilibrium or due to phase transitions. The constant on the right hand side of $R(t) T(t)$ equation has to be then re-set by calculating the entropy produced in such events.

This formulation is sufficient for studying most of Cosmology. However if we are familiar with Einstein's theory the above laws can be derived systematically. We need to calculate the Einstein tensor. The components of the Ricci tensor and the scalar curvature $R$ in terms of the scale factor 
$R(t)$ are

$$
\begin{aligned}
R_{00} & =-3 \frac{\ddot{R}}{R} \\
R_{i j} & =-6\left(\frac{\ddot{R}}{R}+\frac{\dot{R}^{2}}{R^{2}}+\frac{k}{R^{2}}\right) g_{i j} \\
R & =-6\left(\frac{\ddot{R}}{R}+\left(\frac{\dot{R}}{R}\right)^{2}+\frac{k}{R^{2}}\right)
\end{aligned}
$$

The resulting Einstein equations contain only two non-trivial equations, the $G_{00}$ component and the $G_{i i}$ component where $i=1,2,3$ is any of the space components.

$$
\begin{aligned}
& 00:\left(\frac{\dot{R}}{R}\right)^{2}+\frac{k}{R^{2}}=\frac{8 \pi}{3} G \rho \\
& \text { ii } \quad: \quad 2 \frac{\ddot{R}}{R}+\left(\frac{\dot{R}}{R}\right)^{2}+\frac{k}{R^{2}}=-8 \pi G p
\end{aligned}
$$

It turns out that the $i$ equation can be obtained by differentiating the 00 equation and using the thermodynamic relation

$$
d\left(\rho R^{3}\right)=-p d\left(R^{3}\right)
$$

Hence we only state the second law rather than the $i$ equation.

\subsection{Example : Friedman Universe}

An early model due to A. A. Friedman (1922) considers a $k=1$ universe with pressureless dust, i.e., $p=0$ and with $\Lambda=0$. Then according to Law 2,

$$
\frac{d}{d t}\left(\rho R^{3}\right)=0
$$

Now let $t_{1}$ be a reference time so that for any other time $t, \rho(t) R^{3}(t)=\rho\left(t_{1}\right) R^{3}\left(t_{1}\right)$ Then according to Law 1 ,

$$
\begin{aligned}
\frac{1}{R^{2}}\left(\frac{d R}{d t}\right)^{2}+\frac{k}{R^{2}} & =\frac{8 \pi}{3} G \rho_{1} \frac{R_{1}^{3}}{R^{3}} \\
\Longrightarrow \dot{R}^{2}-\frac{R_{\max }}{R} & =-1
\end{aligned}
$$

where

$$
R_{\text {max }} \equiv \frac{8 \pi}{3} G \rho_{1} R_{1}^{3}
$$

This equation is first order in time, but non-linear. It can be solved by expressing both $t$ and $R$ in terms of another parameter $\eta$. The solution is

$$
R(\eta)=\frac{1}{2} R_{\max }(1-\cos \eta) \quad t(\eta)=\frac{1}{2} R_{\max }(\eta-\sin \eta)
$$

It results in a shape called cycloid. The first order equation thus solved can also be thought of as a particle in potential $V(x)=-\frac{x_{\max }}{x}$ with total energy -1 .

Exercise : ( Friedman 1924) Solve for the scale factor of a $\Lambda=0$ universe with pressureless dust but with negative constant curvature, i.e., $k=-1$. 


$\begin{array}{ll}\text { Age } t_{0} & 13.7 \pm 0.2 \mathrm{G} . \mathrm{yr}, \\ \text { Hubble constant } H_{0} & h \times 100 \mathrm{~km} / \mathrm{s} / \mathrm{Mpc} \\ \text { Parameter } h & 0.71 \text { or } 0.73 \\ T_{C M B} & 2.725 \times 10^{6} \mu \mathrm{K} \\ \Omega & 1.02 \pm 0.02 \\ \Omega_{\text {all matter }} h^{2} & 0.120 t o 0.135 \\ \Omega_{b} h^{2} & 0.022 \\ \Omega_{\Lambda} & 0.72 \pm 0.04 \\ w(\equiv p / \rho) & -0.97 \pm 0.08\end{array}$

\subsection{Parameters of the Universe}

We now summarise the observable parameters of the Universe as available from several different data sets. But first we introduce some standard conventions. First we rewrite the first law including the cosmological constant,

$$
H^{2}+\frac{k}{R^{2}}-\frac{\Lambda}{3}=\frac{8 \pi G}{3} \rho
$$

Suppose have a way of measuring each of the individual terms in the above equation. Then with all values plugged in, the left hand side must balance the right hand side. Our knowledge of the Hubble constant $H_{0}$ is considerably more accurate than our knowledge of the average energy density of the Universe. We express the various contributions in the above equation as fractions of the contribution of the Hubble term. This can of course be done at any epoch. Thus, dividing out by $H^{2}$,

$$
1+\frac{k}{H^{2} R^{2}}=\Omega_{\Lambda}+\Omega_{\rho}
$$

where we define the fractions

$$
\Omega_{\Lambda}=\frac{\Lambda}{3 H^{2}} \quad \Omega_{\rho}=\frac{8 \pi G \rho}{3 H^{2}}
$$

In detail, due to several different identifiable contributions to $\rho$ from baryons, photons and Dark Matter, we identify individual constributions again as fractions of the corresponding Hubble term as $\Omega_{b}, \Omega_{\gamma}$, and $\Omega_{D M}$ respectively. Either the sum of the various $\Omega$ 's must add up to unity or the $k \neq 0$. In table 3.2 we list the current values of the parameters.

Determination of Hubble constant has several problems of calibration. It is customary to treat its value as an undetermined factor $h$ times a convenient value $100 \mathrm{~km} / \mathrm{s} / \mathrm{Mpc}$ which sets the scale of the expansion rate. It is customary to state many of the parameters with factors of $h$ included since they are determined conditionally. At present $h^{2} \approx 1 / 2$. The Dark Energy component seems to behave almost like a cosmological constant and hence its contribution is given the subscript $\Lambda$. $\Omega$ being close to unity signifies that we seem to have measured all contributions to energy density needed to account for the observed Hubble constant. The parameter $w$ exactly -1 corresponds to cosmological constant. Current data are best fitted with this value of $w$ and assumption of cold, i.e., non-relativistic Dark Matter. This Friedman model is referred to as the $\Lambda$-CDM model.

\section{The Big Bang Universe}

By its nature as a purely attractive force, gravity does not generically allow static solutions. Since the Universe is isotropic and homogeneous now it is reasonable to assume that that is the way it has been for as far back as we can extrapolate. Such an extrapolation however will require the Universe to have passed through phases of extremely high densities and pressures, phases where various microscopic forces become unscreened long range forces, again subject to overall homogeneity and isotropy of the medium. This is the essential picture of a Big Bang Universe, extrapolated as far back as the Quantum Gravity era about which we can not say much at the 
present state of knowledge. However the intervening epochs encode into the medium important imprints of the possible High Energy forces that may have been operative. Thus the early Universe is an interesting laboratory for Elementary Particle Physics.

\subsection{Thermodynamic Relations}

For a massless single relativistic species,

$$
\begin{aligned}
\rho & =\frac{\pi^{2}}{30} g T^{4} \text { for bosons } & \ldots \times \frac{7}{8} \text { for fermions } \\
n= & \frac{\xi(3)}{\pi^{2}} g T^{3} \text { for bosons } & \ldots \times \frac{3}{4} \text { for fermions }
\end{aligned}
$$

where $g$ is the spin degeneracy factor. In the early Universe, a particle species with mass much less than the ambient temperature behaves just like a relativistic particle. 2 . More generally, we introduce an effective degeneracy $g_{*}$ for a relativistic gas which is a mixture of various species,

$$
\begin{aligned}
& \rho^{\text {relat }}=\frac{\pi^{2}}{30} g_{*} T^{4} \equiv 3 p^{\text {relat }} \\
& g_{*}=\sum_{i} g_{i}\left(\frac{T_{i}}{T}\right)^{4}+\frac{7}{8} \sum_{j} g_{j}\left(\frac{T_{j}}{T}\right)^{4} \\
& \text { Bose Fermi } \\
& \text { species species }
\end{aligned}
$$

As $T$ continues to drop species with rest masses $m_{i} \gg T$ become nonrelativistic and stop contributing to the above. For such species,

$$
\begin{aligned}
\rho_{i}^{\text {non-relat. }} & =m_{i} n_{i} \\
& =\frac{m_{i}\left(n_{i}\left(t_{1}\right) R^{3}\left(t_{1}\right)\right)}{R^{3}(t)} \\
p_{i}^{\text {non-relat }} & =0 \rightarrow \text { "dust" }
\end{aligned}
$$

\subsection{Isentropic Expansion}

We assume particle physics time scales to remain far shorter than expansion time scale $H(t)^{-1}$. Thus we have approximate equilibrium throughout and we have been using equilibrium thermodynamics. Specifically entropy is conserved. While the usual term for such a system is adiabatic, the term in the sense of being isolated from "other" systems does not apply to the Universe and we shall refer directly to fact that entropy is conserved. With no other system with which to exchange energy, it can be shown for the primordial contents of the Universe, that (Kolb \& Turner, Section $3.4)$

$$
d\left[\frac{(\rho+p) V}{T}\right]=0=d S
$$

\footnotetext{
${ }^{2}$ Massless vector bosons have one degree of freedom less than massive vector bosons. So this has to be accounted for in some way. In the Standard Model of Particle Physics, masses arise from spontaneous symmetry breaking which disappears at sufficiently high temperature and the longitudinal vector boson degree of freedom is recovered as additional scalar modes of the Higgs at high temperature.
} 
we define $s \equiv \frac{S}{V}=\frac{\rho+p}{T}$ which is dominated by contribution of relativistic particles

$$
s=\frac{2 \pi^{2}}{45} g_{* s} T^{3}
$$

with

$$
\begin{gathered}
g_{* s}=\sum_{i} g_{i}\left(\frac{T_{i}}{T}\right)^{3}+\frac{7}{8} \sum_{j} g_{j}\left(\frac{T_{j}}{T}\right)^{3} \\
\text { Bose }
\end{gathered}
$$

Note that this is a different definition of the effective number of degrees of freedom than in the case of energy density $\rho$.

\subsection{Temperature Thresholds}

While the conditions of approximate equilibrium and isentropic expansion hold for the most part, crucial energy thresholds in microphysics alter the conditions in the early Universe from time to time and leave behind imprints of these important epochs. Based on the scales of energy involved, we present here a short list of important epochs in the history of the early Universe

$$
\begin{aligned}
& T \quad \text { Relevant species } g_{*} \\
& T \ll \mathrm{MeV} \quad 3 \nu \text { 's; photon }(\gamma) \quad 3.36 \text { (see reason below) } \\
& 1 \mathrm{MeV} \text { to } 100 \mathrm{MeV} \quad 3 \nu \text { 's; } \gamma ; e^{+} e^{-} \quad 10.75 \\
& \geq 300 \mathrm{GeV} \quad 8 \text { gluons, } 4 \text { electroweak gauge } 106.75 \\
& \text { bosons; quarks \& leptons (3 gen- } \\
& \text { erations), Higgs doublet }
\end{aligned}
$$

Above $300 \mathrm{GeV}$ scale, no known particles are non-relativistic, and we have the relations

$$
\begin{aligned}
H & =1.66 g_{*}^{1 / 2} \frac{T^{2}}{M_{P l}} \\
t & =0.30 l g_{*}^{1 / 2} \frac{M_{P l}}{T^{2}} \sim\left(\frac{T}{M e V}\right)^{-2} \sec
\end{aligned}
$$

where

$$
G=\frac{1}{\left(M_{P l}\right)^{2}}=\frac{1}{\left(1.2211 \times 10^{19} \mathrm{GeV}\right)^{2}}
$$

\subsection{Photon Decoupling and Recombination}

We now see in greater detail how one can learn about such thresholds, with the example of photons. As the primordial medium cools, at some epoch, neutral hydrogen forms and photons undergo only elastic (Thomson) scattering from that point onwards. Finally photons decouple from matter when their mean free path grows larger than Hubble distance $H^{-1}$.

$$
\Gamma_{\gamma}=n_{e} \sigma_{T}<H
$$

Here $\sigma_{T}=6.65 \times 10^{-25} \mathrm{~cm}^{2}$ is the Thomson cross-section. (Verify that in $\mathrm{eV}$ units, $\sigma_{T}=1.6 \times$ $\left.10^{-4}(\mathrm{MeV})^{-2}\right)$. Note that we should distinguish this event from the process of "recombination", which is the formation of neutral Hydorgen. In this case the competition is between cross section for ionisation and the expansion rate of the Universe. By contrast the above relation determines the epoch of decoupling of photons, also sometimes called "the surface of last scattering". Thus the process of recombination ends with residual ionisation which is the $n_{e}$ required above to determine decoupling. 


\subsubsection{Saha Equation for Ionization Fraction}

To put above condition to use, we need $n_{e}$ as a function of time or temperature and then $H$ at the same epoch. $n_{e}$ should be determined from detailed treatment of non-equilibrium processes using Boltzmann equations, which we shall take up later. However, utilizing various conservation laws, we can obtain a relationship between the physical quantities of interest, as was done by Saha first in the context of solar corona.

We introduce the number densities $n_{H}, n_{p}, n_{e}$ of neutral Hydrogen, protons and neutrons respectively. The amount of Helium formed is relatively small, $n_{H e} \simeq 0.1 n_{p}$ and is ignored. Charge neutrality requires $n_{e}=n_{p}$, and Baryon number conservation requires $n_{B}=n_{H}+n_{p}$. From approximate thermodynamic equilibrium, we expect these densities to be determined by the Boltzmann law, where $i$ stands for $H, p$ or $e$ :

$$
n_{i}=g_{i}\left(\frac{m_{i} T}{2 \pi}\right)^{3 / 2} \exp \left(\frac{\mu_{i}-m_{i}}{T}\right)
$$

with $g_{i}$ degeneracy factors, $m_{i}$ the relevant masses and $\mu_{i}$ the relevant chemical potentials respectively. Due to chemical equilibrium,

$$
\mu_{H}=\mu_{e}+\mu_{p}
$$

Thus we can obtain a relation

$$
n_{H}=\frac{g_{H}}{g_{p} g_{e}} n_{p} n_{e}\left(\frac{m_{e} T}{2 \pi}\right)^{-3 / 2}\left(\frac{m_{H}}{m_{p}}\right)^{3 / 2} \exp \left(\frac{B}{T}\right)
$$

where $B$ denotes the Hydrogen binding energy, $B=m_{p}+m_{e}-m_{H}=13.6 \mathrm{eV}$. Finally we focus on the fraction $X_{e} \equiv \frac{n_{p}}{n_{B}}$ of charged baryons relative to total baryon number,

$$
\frac{1-X_{e}^{e q}}{\left(X_{e}^{e q}\right)^{2}}=\frac{4 \sqrt{2}}{\sqrt{\pi}} \xi(3)\left(\frac{n_{B}}{n_{\gamma}}\right)\left(\frac{T}{m_{e}}\right)^{3 / 2} \exp \left(\frac{B}{T}\right)
$$

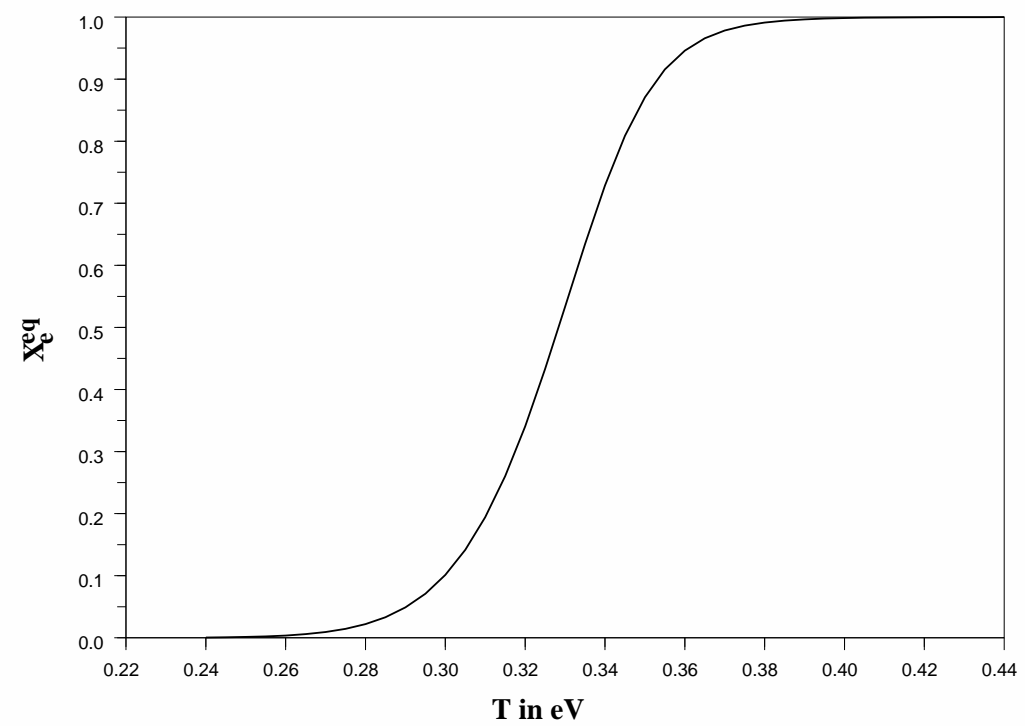

Figure 1: Ionization fraction as a function of photon temperature expressed in $\mathrm{eV}$ 
Fig. 11 shows variation of $X_{e}^{e q}$ as function of the photon temperature $T$, using the value of $n_{B} / n_{\gamma}=6 \times 10^{-10}$ as known from Big Bang Nucleosynthesis calculations and WMAP data. We see that the ionization fraction reduces to $5 \%$ when $T \approx 0.29 \mathrm{eV} \approx 350 \mathrm{~K}$. These are our estimates of the ionisation fraction (set arbitrarily at $5 \%$ ) and the temperature of the last scattering.

Using this estimate we can now calculate the Hubble parameter in the inequality which determines decoupling. We assume that the Universe was already matter dominated by this epoch. Then the scale factor obeys

$$
R(t)=\frac{R_{0}}{t_{0}^{2 / 3}} t^{2 / 3}
$$

where $t_{0}$ and $R_{0}$ are a particular epoch and the corresponding scale factor, chosen here to be the current values signified by subscript 0 . Using this we try to determine the $H$ at decoupling epoch in terms of $H_{0}$.

$$
\frac{\dot{R}}{R}=\frac{2}{3 t}=\frac{H}{H_{0}} H_{0}=\left(\frac{\rho}{\rho_{0}}\right)^{1 / 2} H_{0}=\left(\frac{n_{B}}{n_{B 0}}\right)^{1 / 2} H_{0}
$$

Combine this with the relation

$$
\Omega_{B}=\frac{\rho_{B 0}}{\rho_{\text {crit0 }}}=\frac{m_{p} n_{B 0}}{3 H_{0}^{2} / 8 \pi G}
$$

where $m_{p}$ is the proton mass. Then the condition for photon decoupling becomes

$$
X_{e}^{e q} \sigma_{T}<\left(\frac{T_{0}}{T}\right)^{3 / 2} \frac{8 \pi}{3} G \frac{m_{p}}{\Omega_{B} H_{0}}
$$

Substituting $\sigma_{T}$ expressed in eV units computed above, and $X_{e}^{e q}$ of $5 \%$ and the corresponding temperature found from the graph of Saha formula, we can check that the temperature of the photons decoupled at that epoch should today be

$$
T_{0} \sim(32 K) \times\left(\Omega_{B}\right)^{2 / 3}
$$

Although very crude, this estimate is pretty close to the order of magnitude of the temperature of the residual photons today. We have thus traced a possible way Alpher and Gamow could anticipate the presence of residual radiation from the Big Bang at approximately such a temeperature.

\section{Phase Transitions with Quantum Fields in the Early Uni- verse}

We have been considering an expanding Universe, but its expansion rate is so slow that for most of its history, it is quasi-static as far as Particle Physics processes are concerned. Under this assumption, we can think of a thermal equilibrium for substantial periods on the microscopic scale, and build in the slow evolution of the scale factor as a significant but separate effect.

Quantized fields which are systems of large numbers of degrees of freedom display different collective behavior, with qualitative changes in ground state as a function of temperature. The technique for studying the ground state behavior at non-zero temperature is a modification of the process of calculating Green functions using path integral method in functional formalism.

\subsection{Legendre Transform}

As a warm up for the method to be used, consider a magnetized spin system with spin degrees of freedom $s(x)$ as a function of position, and $\mathcal{H}$ the Hamiltonian of the system. If we want to determine the equilibrium value of magnetization, we first introduce an external field $H$, in the presence of which, the Helmholtz Free Energy $F$ is given by

$$
Z(H) \equiv e^{-\beta F(H)}=\int \mathcal{D} s \exp \left(-\beta \int d x \mathcal{H}(s)-H s(x)\right)
$$


where $\beta=1 / T$, (same as $1 /(k T)$ in our units) and $\mathcal{D} s$ denotes functional integration over $s(x)$. Now the quantity of interest is

$$
M=\int d x\langle s(x)\rangle=\frac{1}{\beta} \frac{\partial}{\partial H} \log Z=-\frac{\partial F}{\partial H}
$$

We now introduce a function of $M$ itself, the Gibbs free energy, through the Legendre transform

$$
G(M)=F+M H
$$

so that by inverse transform,

$$
H=\frac{\partial G}{\partial M}
$$

Now $H$ being an auxiliary field has to be set to zero. Thus the last equation can be now read as follows. The equilibrium value of $M$ can be found by minimizing the function $G(M)$ with respect to $M$. In other words, for studying the collective properties of the ground state, $G$ is the more suitable object than $\mathcal{H}$.

\subsection{Effective action and effective potential}

In Quantum Field Theory, a similar formalism can be set up to study the collective behavior of a bosonic field $\phi$. It is possible in analogy with the above, to define a functional $\Gamma$ of an argument suggestively called $\phi_{c l}$ designating the c-number or the classical value of the field. Analgous to the external field $H$ above, an auxiliary external current $J(x)$ is introduced. Then

$$
Z[J] \equiv e^{-i E[J]}=\int \mathcal{D} \phi \exp \left[i \int d^{4} x(\mathcal{L}[\phi]+j \phi)\right]
$$

Then we obtain the relations

$$
\begin{aligned}
\frac{\delta E}{\delta J}=i \frac{\delta \log Z}{\delta J(x)} & =-\langle\Omega|\phi(x)| \Omega\rangle_{J} \\
& \equiv \phi_{c l}(x)
\end{aligned}
$$

Therefore, let

so that

$$
\Gamma\left[\phi_{c l}\right] \equiv-E[J]-\int d^{4} y J(y) \phi_{c l}(y)
$$

$$
\frac{\delta}{\delta \phi_{c l}} \Gamma\left[\phi_{c l}\right]=-J(x)
$$

Thus the quantum system can now be studied by focusing on a classical field $\phi_{c l}$, whose dynamics is determined by minimizing the functional $\Gamma$. The auxiliary current $J$ is set zero at this stage. This is exactly as in classical mechanics, minimizing the action for finding Euler-Lagrange equations. The functional $\Gamma$ is therefore called the effective action. If it can be calculated, it captures the entire quantum dynamics of the field $\phi$ expressed in terms of the classical function $\phi_{c l}$.

Calculating $\Gamma$ can be an impossible project. A standard simplification is to demand a highly symmetric solution. If we are looking for the properties of a physical system which is homogeneous and in its ground state, we need the collective behavior of $\phi$ in a state which is both space and time translation invariant. In this case $\phi_{c l}(x) \rightarrow \phi_{c l}, \partial_{\mu} \phi_{c l}=0$ and $\Gamma$ becomes an ordinary function (rather than a functional) of $\phi_{c l}$. It is now advisable to factor out the spacetime volume to define

$$
V_{e f f}\left(\phi_{c l}\right)=-\frac{1}{(V T)} \Gamma\left[\phi_{c l}\right]
$$

so that the ground state is given by one of the solutions of

$$
\frac{\partial}{\partial \phi_{c l}} V_{e f f}\left(\phi_{c l}\right)=0
$$


In general $V_{\text {eff }}$ can have several extrema. The minimum with lowest value of $V_{e f f}$, if it is unique, characterises the ground state while the other minima are possible metastable states of the system. A more interesting case arises when the lowest energy minimum is not unique. In a quantum system with finite number of degrees of freedom, this would not result in any ambiguity. The possibility of tunneling between the supposed equivalent vacua determines a unique ground state - as in the example of ammonia molecule. But in an infinite dimensional system, such as a field system, we shall see that such tunneling becomes prohibitive, and the energetically equivalent vacua can exist simultaneously as possible ground states.

When we speak of several local minima of $V_{\text {eff }}$ and therefore maxima separating them we are faced with a point of principle. Any function defined as a Legendre Transform can be shown to be intrinsically convex, i.e., it can have no maxima, only minima. The maxima suggested by above extremization process have to be replaced by a construction invented by Maxwell for thermodynamic equilibria. Consider two minima $\phi_{1}$ and $\phi_{2}$ separated by a maximum $\phi_{3}$ as shown in 2. We ignore the part of the graph containing point $\phi_{3}$ as of no physical significance, and introduce a parameter $x$ which permits continuous interpolation between the two minima. Over the domain intermediate between $\phi_{1}$ and $\phi_{2}$, we introduce a parameter $x$ and redefine $\phi_{c l}$ to be

$$
\phi_{c l}=x \phi_{1}+(1-x) \phi_{2} \quad 0 \leq x \leq 1
$$

The assumption is that the actual state of the system is no longer translation invariant and is an

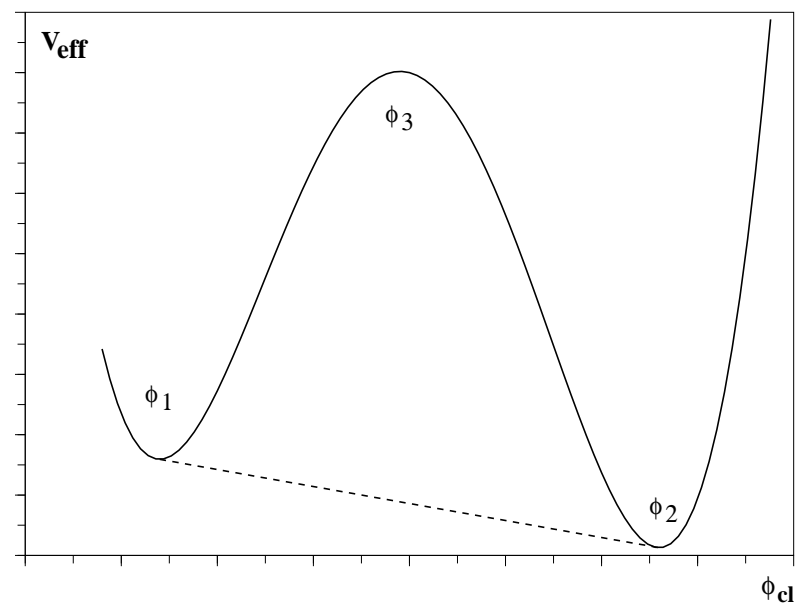

Figure 2: The effective potential of a system with local minimum at $\phi_{1}$ and $\phi_{2}$. The mostly concave segment with the point $\phi_{3}$ is treated as unphysical and replaced by the dashed line.

admixture of phases with either a value $\phi_{1}$ or a value $\phi_{2}$. The above redefinition is interpreted as a value of $\phi$ averaged over such regions. Then if the physical value of $\phi_{c l}$ is characterised by parameter value $x$, we define the corresponding value of $V_{\text {eff }}$

$$
V_{\text {eff }}^{a v g}\left(\phi_{c l}\right)=x V_{\text {eff }}\left(\phi_{1}\right)+(1-x) V_{\text {eff }}\left(\phi_{2}\right)
$$

These formulae will not be of direct relevance to us. However we should remember that while the minima $\phi_{1}$ and $\phi_{2}$ represent possible physical situations, the extremum does not. We shall see that the admixture phase arises when the system begins to tunnel from one vacuum to another due to energetic considerations. We shall develop the formalism for the tunneling process and will be more interested in the tunneling rate which in turn is indicative of how quickly the transition from a false vacuum (a local minimum) to a true vacuum (absolute minimum) can be completed. 


\subsection{Computing $V_{\text {eff }}$}

A very simple example of a non-trivial effective potential is given by a complex scalar field theory with a non-trivial self-coupling

$$
\mathcal{L}=\partial_{\mu} \phi^{\dagger} \partial^{\mu} \phi-\frac{\lambda}{4}\left(\phi^{\dagger} \phi-\mu^{2}\right)^{2}
$$

Note that the mass-squared is negative and so the kinetic terms without the $\phi^{4}$ interaction would imply tachyonic excitations. The correct field theoretic interpretation is to work around a stable minimum such as $\phi=\mu$. Thus we define $\phi(x)=\mu+\tilde{\phi}(x)$ and quantize only the $\tilde{\phi}(x)$ degrees of freedom. By substituting the redefined $\phi$ into the above Lagrangian we find that the field $\tilde{\phi}$ has a mass-squared $+\mu^{2}$ and can be treated perturbatively.

In this example the polynomial $\frac{\lambda}{4}\left(\phi^{\dagger} \phi-\mu^{2}\right)^{2}$ serves as the zeroth order effective potential. The values $\mu e^{i \alpha}$ for real values of alpha between 0 and $2 \pi$ are all permissible vacuum expectation values (VEVs) as determined from minimizing this polynomial. These values are modified in Quantum Field Theory, however in this simple example their effect will only be to shift the value $\mu$ of $|\phi|$ by corrections of order $\hbar$. This example is the well known Goldstone mechanism of symmetry breaking. Here the symmetry breaking is indicated by the classical $V$ itself.

There are important exceptions to this, where quantum corrections become important and they must be computed. One is where the above Lagrangian is modified as follows. Remove the mass term altogether, but couple the massless field to a $U(1)$ gauge field, in other words massless scalar QED. The classical minimum of the potential $\left(\phi^{\dagger} \phi\right)^{2}$ is $\phi=0$. It was shown by S. Coleman and E. Weinberg that after the one-loop quantum effects are taken into account, the minimum of the scalar potential shifts away from zero. The importance of this result is that the corrected vacuum does not respect $U(1)$ gauge invariance. The symmetry breakdown is not encoded in classical $V$ and has to be deduced from the $V_{e f f}$.

Another important example is effects of a thermal equilibrium. Consider the complex scalar of our first example, with mass-squared negative at classical level. Suppose we couple this field to a gauge field. This would lead to spontaneous but classically determinable breakdown of the gauge invariance according to Higgs-Kibble mechanism. If we now include temperature corrections, then in the high temperature limit the minimum of the scalar field Lagrangian in fact shifts to $\phi=0$, thus restoring the symmetry! This is the case important to the early Universe where Universe cools slowly from very high values of temperature, possibly close to the Planck scale. Since Higgs mechanism is the chief device for constructing Grand Unified Theories (GUTs) we find that gauge symmetries are effectively restored at high temperatures and non-trivial vacua come into play only at lower temperatures.

We will be unable to discuss the formalism for computing the effective potential in any detail. However here we shall briefly recapitulate the recipe which reduces the problem to that of computing Feynman diagrams. Recall that an important consequence of quantum corrections can be change in the vacuum expectation value of a scalar field. We shall assume that the vacuum continues to be translation invariant, so that this shifted value is a constant. Anticipating this, in the Lagrangian we shift the field value $\phi(x)=\phi_{c l}+\eta(x)$ where the significance of the subscript $c l$ becomes clear due to the reasoning given above. However note that at his stage $\phi_{c l}$ is only a parameter and the $V_{e f f}$ is computed as a function of it. Now in the presence of an external source $J(x)$,

$$
\begin{aligned}
\int d^{4} x(\mathcal{L}+J \phi) & =\int d^{4} x\left(\mathcal{L}+J \phi_{d}\right)+\int d^{4} x \eta\left(\frac{\delta \mathcal{L}}{\delta \phi}+J\right) \\
& +\frac{1}{2} \int d^{4} x d^{4} y \eta \eta \frac{\delta^{2} \mathcal{L}}{\delta \phi \delta \phi} \ldots
\end{aligned}
$$

Here onwards we assume that the $\phi_{c l}$ is chosen to satisfy

$$
\left.\frac{\delta \mathcal{L}}{\delta \phi}\right|_{\phi=\phi_{c l}}=-J(x)
$$


Note that this $\phi_{c l}$ is still dependent on the external function $J(x)$ and hence adjustable. Then in the path integral formula for the generating functional, we can carry out a saddle point evaluation of the gaussian 3 integral around the extremum defined by the choice of $\phi_{c l}$ just made.

$$
\begin{aligned}
\mathcal{Z}_{J} & =\int \mathcal{D} \eta \exp \left(i \int \mathcal{L}\left(\phi_{c l}\right)+J \phi_{c l}+\frac{1}{2} \int \eta \mathcal{L}^{\prime \prime} \eta\right) \\
& =\exp \left[i \int \mathcal{L}\left(\phi_{c l}\right)+J \phi_{c l}\right] \times\left(\operatorname{det}\left[-\frac{\delta^{2} \mathcal{L}}{\delta \phi \delta \phi}\right]\right)^{-1 / 2} \mathcal{Z}_{2}
\end{aligned}
$$

where the $\mathcal{Z}_{2}$ denotes all the terms of order higher in the variations of $\mathcal{L}$ with respect to $\phi$. In perturbative interpretation hese higher derivatives

$$
\frac{\delta^{n} \mathcal{L}}{\delta \phi \ldots \delta \phi_{n}}
$$

are treated as vertices, while

$$
-i\left(\frac{\delta^{2} \mathcal{L}}{\delta \phi \delta \phi}\right)^{-1}
$$

is used as propagator. The main correction to one-loop order however can be determined directly from the determinant resulting from saddle point integration. While calculating $S$-matrix elements this determinant is only an overall constant and of no significance. But in the effective potential formalism it provides the main correction. It also requires proof to know that the determinant provides the leading correction and that everything inside $\mathcal{Z}_{2}$ represents higher order quantum corrections. This will not be pursued here. We now quote an example.

\subsubsection{An example}

Consider the theory of two real scalar fields $\phi_{1}$ and $\phi_{2}$

$$
\mathcal{L}=\frac{1}{2} \sum_{i}\left(\partial_{\mu} \phi^{i}\right)^{2}+\frac{1}{2} \mu^{2} \sum_{i}\left(\phi^{i}\right)^{2}-\frac{\lambda}{4}\left[\sum_{i}\left(\phi^{i}\right)^{2}\right]^{2}
$$

with $i=1,2$ and $\mu^{2}>0$. The latter condition means that the classical minimum of the theory is not at $\phi_{i}=0$ but at any of the values defined by $\lambda\left(\phi_{1}^{2}+\phi_{2}^{2}\right)=\mu^{2}$. A possible minimum is at $\left(\phi_{1}, \phi_{2}\right)=(\mu / \sqrt{\lambda}, 0)$. If we shift the fields by choosing $\left(\phi_{1}, \phi_{2}\right)=\left(\phi_{c l}+\eta_{1}(x), \eta_{2}(x)\right)$ we get propagators for the two real scalar fields $\eta_{i}$ with mass-squares given by $m_{1}^{2}=3 \lambda \phi_{c l}^{2}-\mu^{2}$ and $m_{2}^{2}=\lambda \phi_{c l}^{2}-\mu^{2}$.

Evaluation of the determinants of the inverse propagators requires a series of mathematical tricks.

$$
\begin{aligned}
\log \operatorname{det}\left(\partial^{2}+m^{2}\right) & =\operatorname{Tr} \log \left(\partial^{2}+m^{2}\right) \\
& =\sum_{k} \log \left(-k^{2}+m^{2}\right) \\
& =(V T) \int \frac{d^{4} k}{(2 \pi)^{4}} \log \left(-k^{2}+m^{2}\right)
\end{aligned}
$$

The determinant actually has a diagrammatic interpretation as was explained by Coleman and Weinberg. Consider a single closed loop formed by joining together $n$ massless propagators, joined together by consecutive mass insertions. Here we are treating treating mass as an interaction for convenience. This loop has $\frac{1}{n !}$ in front of it from perturbation theory rules due to $n$ mass

\footnotetext{
${ }^{3}$ Actually "pseudo"-gaussian due to the presence of $i$, but equivalently after choosing to work with euclidian path integral.
} 
insertions. But there are $(n-1)$ ! ways of making these identical mass isertions. This makes the contribution of this loop weighed by $\frac{1}{n}$. Further because the propagators are identical, $n$th term is $n$th power of first term. Thus summing all the terms with single loop but all possible mass insertions amounts to a log series. The next important argument is that indeed the perturbative expansion is an expansion ordered by the number of loops. By including all contributions at one loop, we have captured the leading quantum correction.

We now turn to evaluation of this using dimensional regularization prescription

$$
\int \frac{d^{d} k}{(2 \pi)^{d}} \log \left(-k^{2}+m^{2}\right)=\frac{-i \Gamma\left(-\frac{d}{2}\right)}{(4 \pi)^{d / 2}} \frac{1}{\left(m^{2}\right)^{-d / 2}}
$$

Therefore,

$$
\begin{aligned}
V_{e f f}\left(\phi_{c l}\right)= & -\frac{1}{2} \mu^{2} \phi_{c l}^{2}+\frac{\lambda}{4} \phi_{c l}^{4} \\
& -\frac{1}{2} \frac{\Gamma(-d / 2)}{(4 \pi)^{d / 2}}\left[\left(\lambda \phi_{c l}^{2}-\mu^{2}\right)^{d / 2}+\left(3 \lambda \phi_{c l}^{2}-\mu^{2}\right)^{d / 2}\right] \\
& +\frac{1}{2} \delta_{\mu} \phi_{c l}^{2}+\frac{1}{4} \delta_{\lambda} \phi_{c l}^{4}
\end{aligned}
$$

where the $\delta_{\mu}$ and $\delta_{\lambda}$ represent finite parts. Now

$$
\begin{aligned}
\frac{\Gamma(2-d / 2)}{(4 \pi)^{d / 2}\left(m^{2}\right)^{2-d / 2}=} & \frac{1}{(4 \pi)^{2}}\left(\frac{2}{\epsilon}-y+\log 4 \pi-\log m^{2}\right) \\
& \stackrel{\overline{\mathrm{MS}} \text { scheme }}{\longrightarrow} \frac{1}{(4 \pi)^{2}}\left(-\log \frac{m^{2}}{M^{2}}\right)
\end{aligned}
$$

where a reference mass scale $M$ has to be introduced. Therefore, with superscript (1) signifying one-loop correction,

$$
\begin{aligned}
V_{\text {eff }}^{(1)}= & \frac{1}{4} \frac{1}{(4 \pi)^{2}}\left[\left(\lambda \phi_{c l}^{2}-\mu^{2}\right)^{2}\left(\log \left(\lambda \phi_{c l}^{2}-\mu^{2}\right) / M^{2}-3 / 2\right)\right] \\
& +\left(3 \lambda \phi_{c l}^{2}-\mu^{2}\right)^{2}\left(\log \left[\left(3 \lambda \phi_{c l}^{2}-\mu^{2}\right) / M^{2}\right]-3 / 2\right)
\end{aligned}
$$

A rule of thumb summary of this example is that the one-loop correction to the effective potential is $\left(1 / 64 \pi^{2}\right) m_{e f f}^{4} \ln \left(m_{e f f} / M\right)^{2}$ where $m_{e f f}^{2}$ contains $\phi_{c l}^{2}$ and $M$ is a reference mass scale required by renormalization. Further, it can be shown that the modification to the tree level (classical) vacuum expectation value comes only from the $m^{4} \ln (m / M)^{2}$ term of the field direction in which the vacuum is already shifted, $\phi_{1}$ in the present example.

\subsection{Temperature corrections to 1-loop}

In the early universe setting we are faced with doing Field Theory in a thermal bath, also referred to as "finite temperature field theory". With some clever tricks and exploiting the analogy of the field theory generating functional with the partition function for a thermal ensemble, one can reduce this problem also to that of calculating an effective potential. The key modification introduced is to combine the time integration of the generating functional and the multiplicative $-\beta$ (inverse temperature) occurring in the partition function into an imaginary time integral $-\int_{0}^{\beta} d \tau$. Further, the trace involved in the thermal averaging can be shown to be equivalent to periodicity in imaginary time of period $\beta$. For bosonic fields one is lead to periodic boundary condition and for fermionic fields one is lead to anti-periodic boundary condition. Thus the usual 
propagator is replaced by an imaginary time propagator of appropriate periodicity. For a scalar field of mass $m$, thermal propagator $\Delta^{T}$ is given by

$$
\Delta^{T}(x, y)=\frac{1}{\beta} \sum_{k^{0}=2 \pi i n / \beta} \int \frac{d^{3} k}{(2 \pi)^{3}} e^{-i k \cdot(x-y)} \frac{i}{k^{2}-m^{2}}
$$

We now quote the result for the temperature dependent effective potential $V^{T}$. We focus only on the $\phi_{1}$ degree of freedom of the previous example and drop the subscript 1 ,

$$
\begin{aligned}
V_{e f f}^{T}\left[\phi_{c l}\right] & =V_{\text {eff }}\left[\phi_{c l}\right]+\frac{T^{4}}{2 \pi^{2}} \int_{0}^{\infty} d x x^{2} \ln \left[1-\exp \left(-\left(x^{2}+\frac{m^{2}}{T^{2}}\right)^{1 / 2}\right)\right] \\
\text { with } m^{2}\left(\phi_{c l}\right) & =-\mu^{2}+3 \lambda \phi_{c l}^{2}
\end{aligned}
$$

and $V_{\text {eff }}$ to one-loop order is as obtained in the previous subsection. In the high temperature limit $T \gg \phi_{c l}$ we can determine the leading effects of temperature by expanding the above expression to find

$$
V_{e f f}^{T}=V_{e f f}+\frac{\lambda}{8} T^{2} \phi_{c l}^{2}-\frac{\pi^{2}}{90} T^{4}+\ldots
$$

From thermodynamic point of view this $V_{\text {eff }}$ represents the Gibbs free energy. Entropy density, pressure and the usual energy density are given by

$$
\begin{aligned}
s\left[\phi_{c l}\right] & =-\frac{\partial V_{e f f}^{T}}{\partial_{T}} ; \quad \text { while } p=-V^{T}\left[\phi_{c l}\right] \\
\operatorname{and} \rho\left[\phi_{c l}\right] & =V_{e f f}^{T}+T s\left[\phi_{c l}\right] \\
& =V\left(\phi_{c l}\right)-\frac{\lambda}{8} T^{2} \phi_{c l}^{2}+\frac{\pi^{2}}{30} T^{4} .
\end{aligned}
$$

The resulting graphs of $V_{e f f}^{T}$ are plotted in fig. 3. In plotting these, the term $T^{4}$ which is the usual thermodynamic contribution, but which is independent of the flield $\phi_{c l}$ is subtracted.

We can summarise the main results of this subsection retaining a single scalar degree of freedom $\phi$ for which,

$$
\mathcal{L}=\frac{1}{2} \partial_{\mu} \phi \partial^{\mu} \phi+\frac{1}{2} \mu^{2} \phi^{2}-\frac{1}{4} \lambda \phi^{4}
$$

with minima at $\sigma_{ \pm}= \pm \sqrt{\mu^{2} / \lambda}$

The leading effect of the temperature correction is to add a term $T^{2} \phi_{c l}^{2}$ so that

$$
V_{e f f}^{T}=\left(-\frac{1}{2} \mu^{2}+\frac{\lambda}{8} T^{2}\right) \phi_{c}^{2}+\frac{1}{4} \lambda \phi_{c}^{4}+\ldots
$$

This is extremized at the values

$$
\phi_{c l}= \pm \sqrt{\frac{\mu^{2}-\lambda T^{2} / 4}{\lambda}} \text { and } \phi_{c l}=0
$$

Further, note that

$$
\left.\frac{\partial^{2} V}{\partial \phi^{2}}\right|_{\phi_{c}=0}=-\mu^{2}+\frac{\lambda}{4} T^{2}
$$

As a result we see that the curvature can change sign at the trivial extremum $\phi_{c l}=0$ at a critical temperature $T_{c}=2 \mu / \sqrt{\lambda}$. 


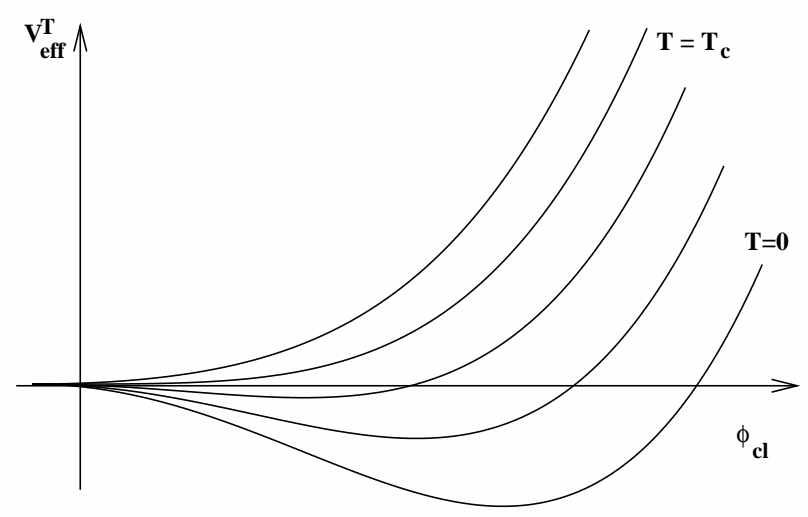

Figure 3: Temperature dependent effective potential plotted to show its dependence on $\phi_{c l}$ for various values of temperature $T . T_{c}$ denotes the temperature below which the trivial minimum is unstable.

\section{First order Phase transition}

At the end of the last section we saw that the minimum at $\phi=0$ can turn into a maximum as the temperature drops below the critical value $T_{c}$. This effect is felt simultaneously throughout the system and a smooth transition to the newly available minimum with $\phi \neq 0$ ensues. The expectation value of $\phi$ is called the order parameter of the phase transition, and in this case where it changes smoothly from one value to another over the entire medium is called a Second Order phase transition.

But there can also be cases where there are several minima of the free energy function but separated by an energy barrier. Thus the system can end up being in a phase which is a local minimum, called the false vacuum, with another phase of lower free energy, called the true vacuum, available but not yet accessed. If the barrier is not too high, thermal fluctuations can cause the system to relax to the phase of lower free energy. The probability for the system to make the transition is expressed per unit volume per unit time and has the typical form

$$
\Gamma=A \exp \{-B\}
$$

The expressions $A$ and $B$ are dependent upon the system under consideration. The presence of $B$ reminds us of the Boltzmann type suppression that should occur if the system has to overcome an energy barrier in the process of making the transition. In Quantum Field Theory there are also quantum mechanical fluctuations which assist this process. We observe that the formula above is also of the type of WKB transition rate in Quantum Mechanics. Indeed, we have partly thermal fluctuations and partly tunneling effects responsible for this kind of transition. A convenient formalism exists for estimating the combined effects using the thermal effective potential introduced in the previous section.

A transition of this type does not occur simultaneously over the entire medium. It is characterized by spontaneous occurrance of small regions which tunnel or fluctuate to the true vacuum. Such regions of spontaneously nucleated true vacuum are called "bubbles" and are enclosed from the false vacuum by a thin boundary called the "wall". Since the enclosed phase is energetically favorable, such bubbles begin to expand, as soon as they are formed, into the false vacuum. Over time such bubbles keep expanding, with additional bubbles continuing to nucleate, and as the 
bubbles meet, they merge, eventually completing the transition of the entire medium to the true vacuum. Such a transition where the order parameter $p h i$ has to change abruptly from one value to another for the transition to proceed is called a First Order phase transition (FOPT).

\subsection{Tunneling}

At first we shall consider tunneling for a field system only at $T=0$. An elegant formalism has been developed which gives the probability per unit volume per unit time for the formation of bubbles of true vacuum of a given size. Consider a system depicted in fig. [ which has a local minimum at value $\phi_{1}$, chosen to be the origin for convenience. There are other configurations of same energy, such as $\phi_{2}$ separated by a barrier and not themselves local minima. If this is an ordinary quantum mechanical system of one variable and the initial value of $\phi$ is $\phi_{1}$, the system is unstable towards tunneling to the point $\phi_{2}$ and subsequently evolving according to usual dynamics. If this were

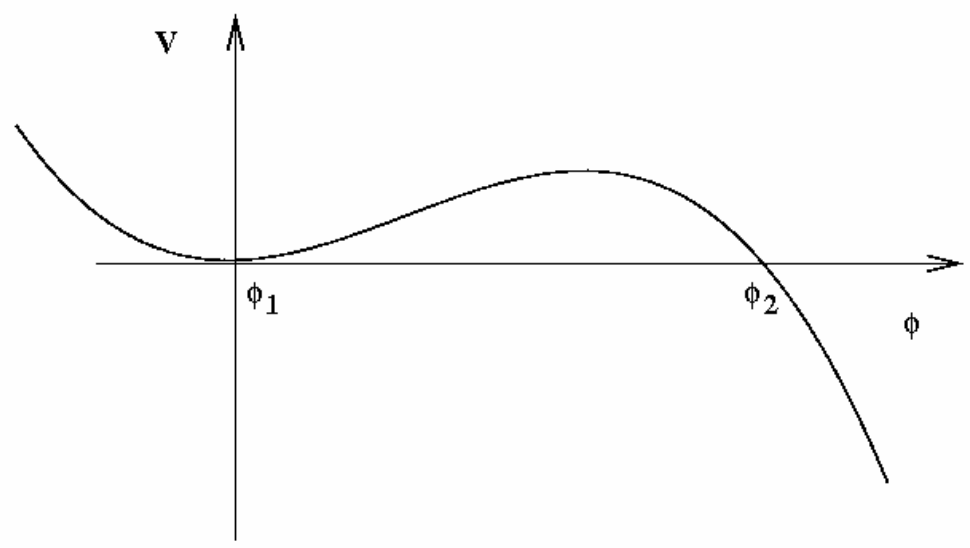

Figure 4: A system which has a local minimum at $\phi_{1}$ and which is unstable towards tunneling to a point $\phi_{2}$ of equal energy

point particle mechanics, the formula for the transition amplitude from the state $\left|\phi_{1}\right\rangle$ to the state $\left|\phi_{2}\right\rangle$ is given in the Heisenberg picture and in the path integral formulation as

$$
\left\langle\phi_{2}\left|e^{-\frac{i}{\hbar} H T}\right| \phi_{1}\right\rangle=\int \mathcal{D} \phi e^{\frac{i}{\hbar} S}
$$

where $T$ is a time interval and the action $S$ on the right hand side has the same range of time integration. Instead of evaluating this directly, we make two observations. Firstly, if we asked for the amplitude for the state $\left|\phi_{1}\right\rangle$ to evolve into itself after time $T$, it would involve contributions also from paths that access the state $\left|\phi_{2}\right\rangle$. Thus if we inserted a complete set of states on the left hand side above at an intermediate time, say, time $T / 2$ (we justify the $T / 2$ later), among the many contributions there would also occur the term

$$
\left\langle\phi_{1}\left|e^{-\frac{i}{\hbar} H T / 2}\right| \phi_{2}\right\rangle\left\langle\phi_{2}\left|e^{-\frac{i}{\hbar} H T / 2}\right| \phi_{1}\right\rangle
$$

Correspondingly on the right hand side we would have contribution from paths that start at the point $\phi_{1}$ and end at $\phi_{1}$, but after reaching $\phi_{2}$ somewhere along the trajectory.

The second point is more interesting. Actually the presence of $\left|\phi_{2}\right\rangle$, a state of equal energy, makes $\left|\phi_{1}\right\rangle$ unstable. Hence the contribution such as $\left\langle\phi_{1}\left|e^{-\frac{i}{\hbar} H T / 2}\right| \phi_{2}\right\rangle$ actually makes the total amplitude for $\phi_{1}$ returning to $\phi_{1}$ smaller than unity in magnitude. This happens only if the evolution operator $e^{-\frac{i}{\hbar} H T / 2}$ somehow departs from being of unit magnitude, i.e., its exponent becomes real negative rather than pure imaginary. 
Thus if we look, not for the entire amplitude, but only for the part where the exponent becomes effectively imaginary then that part of the sum over intermediate states actually indirectly gives the transition amplitude $\left\langle\phi_{2}\left|e^{-\frac{i}{\hbar} H T / 2}\right| \phi_{1}\right\rangle$, the one we started out to look for (aside from factor $1 / 2$ in time). In the limit $T$ becomes infinite, all the contributions with real negative exponents will go to zero. The leading contribution is the term with the smallest exponent. On the right hand side this means that among all the paths that start from one vacuum, sample the other and return, the one that minimises the action will contribute. Again we expect, on the right hand side, the exponent to be real negative, i.e., the contribution of a Euclidean path, with $i \int d t$ replaced by $-\int d \tau$. This is also reasonable since we know the usual kinetic energy of the particle has to be replaced by a negative contribution when the trajectory is under the barrier.

The summary of this discussion is that actually we should be looking only for the imaginary part of the contributions on both the sides of the formula above. If we find the path which minimises the Euclidean action, then in terms of that, to leading order, and in the semi-classical limit, we have the tunneling formula

$$
\Gamma=A \exp \left(-S_{E}\right)
$$

We can also now see the reason for $T / 2$ to be the appropriate time. If the path minimises the action it should be as symmetric as possible. Thus we expect a time symmetry $T \rightarrow-T$ and this explains why the escape point $\phi_{2}$ should occur at $T / 2$. We therefore solve the Euler-Lagrange equations derived from the action

$$
S_{E}=\int d^{4} x\left\{\frac{1}{2}\left(\frac{d \phi}{d \tau}\right)^{2}+\frac{1}{2}|\nabla \phi|^{2}+V[\phi]\right\}
$$

Now the action will be minimum for the path that has the fewest wiggles, i.e., is mostly monotonic. We expect the path to start at large negative $\tau$ at the value $\phi_{1}$ and stay at that value as much as possible, and monotonically reach $\phi_{2}$ near the origin, and then retrace a symmetric path back to $\phi_{1}$ as $\tau$ goes to infinity. Such a path which bounces back has been called "the bounce".

To solve for the bounce, our first simplification will be to invoke space-time symmetries, viz., we assume that the configuration of fields which will minimise the integral in question will obey spatial isotropy. This is same as assuming that the spontaneously formed bubble will be spherically symmetric. With 4 Euclidean dimensions, assuming 0(4) symmetry, one solves the equation

$$
\phi^{\prime \prime}+\frac{3}{r} \phi^{\prime}-V^{\prime}(\phi)=0
$$

One boundary condition is $\phi(r \rightarrow \infty)=\phi_{1}$ where we have chosen $\phi_{1}=0$. At the origin we could place the requirement $\phi(r=0)=\phi_{2}$ but it is more important to require $\phi^{\prime}(r=0)=0$ as is usual for spherical coordinates when the solution is expected to be smooth through the origin. Indeed we may not even know what the "exit point" $\phi_{2}$ after the tunelling will be. The bounce when solved for will also reveal it. A typical bounce solution is shown in figure 5 .

\subsubsection{Next to leading order}

According to above discussion, the tunneling rate is given by a WKB type formula $\Gamma=A \exp \left(-S_{E}\right)$. With $S_{E}\left(\phi_{\text {bounce }}\right)$ determined by extremising the Euclidean action, the exponential is the most important factor in this formula. The front factor $A$ arises from integration over the small fluc-

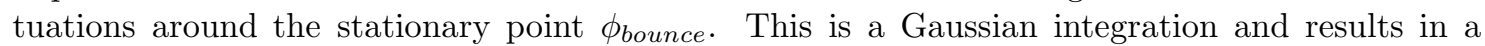
determinant. There are several subtleties which arise. The answer is that we need to remove zero mode(s) of the fluctuation operator, and normalize with respect to the determinant of the fluctuations in the absence of the bounce. Thus with prime on "det" denoting removal of zero $\operatorname{mode}(\mathrm{s})$,

$$
A=\left(\frac{S_{E}(\phi)}{2 \pi}\right)^{2}\left(\frac{\operatorname{det}^{\prime}\left[-\square_{E}+V^{\prime \prime}\left(\phi_{\text {bounce }}\right)\right]}{\operatorname{det}\left[-\square_{E}+V^{\prime \prime}(0)\right]}\right)^{-1 / 2}
$$




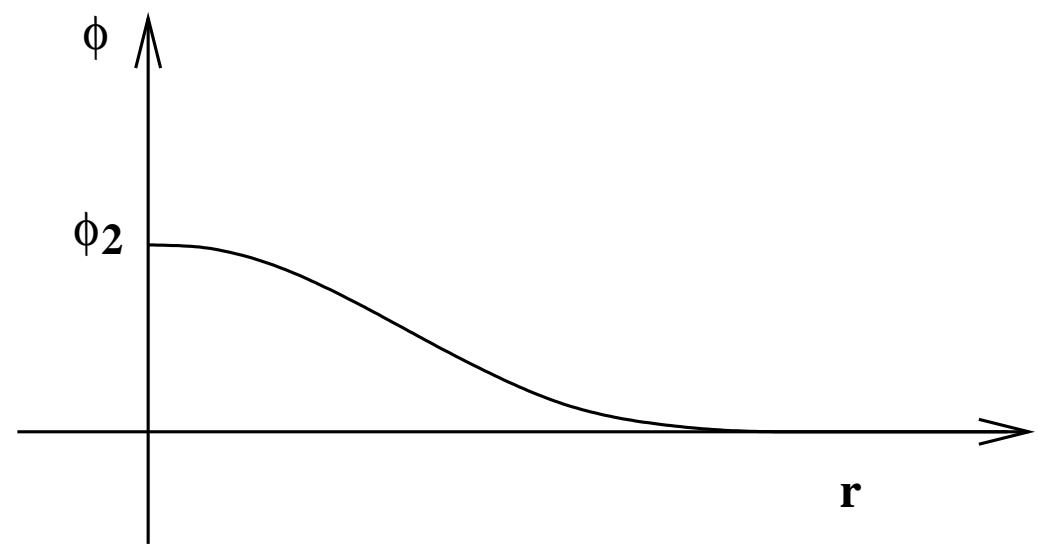

Figure 5: Tunneling rate is determined by the minimum of the Euclidean action $\phi_{\text {bounce }}(r)$ obeying appropriate symmetry and boundary conditions.

\subsubsection{Thermal bounce}

We can now address the tunneling problem in a thermal ensemble, i.e., at $T \neq 0$. Recall our observation during the discussion of thermal effective potential, viz., the analogy between Euclidean path integral and the trace weighed by density matrix in thermal partition function. By the same arguments we can show that we must look for a bounce solution periodic in imaginary time with period $1 / \beta$. There will be an infinite number of such bounces, but the one to dominate will have least number of extrema. Then we obtain the rate formula

$$
\Gamma^{[T]}=A \exp \left(-S_{E}^{[T]}\right)
$$

with

$$
S_{E}^{[T]}=4 \pi \beta \int_{0}^{\infty} r^{2} d r\left[\frac{1}{2} \phi^{2}+V_{e f f}^{T}(\phi)\right]
$$

To obtain the euclidian action in this case, we solve the equations of motion obtained by varying this action and solve them subject to $O(3)$ symmetry of spatial directions. This is called the "bounce" solution relevant to thermal transitions and the corresponding value of the action is inserted into the rate formula.

\subsection{Applications}

The formalism developed in this section is important for determining the evolution of the Universe when the field theory signals a first order phase transition. The rate $\Gamma$ can vary greatly due to the exponential factor. If the rate is too small, the expansion rate of the Universe may be faster and in this case parts of the Universe may never tunnel to the true vacuum. If the state of broken symmetry is phenomenologically desirable, the rate should be fast enough, at least faster than the expansion rate of the Universe at the time of Big Bang Nucleosynthesis (BBN). The end of a first order phase transition dumps a certain amount of entropy into the Universe, similar to latent heat in usual substances. Since the history of the Universe after the BBN is fairly precisely known any unusual phenomenon especially one that may disturb the baryon to entropy ratio should occur well before the BBN and not alter the required value of the ratio.

Such considerations place constraints on the parameters of the scalar field theory undergoing the phase transition. The first proposal of inflationary Universe required a fairly specific range for the value of the rate, slow enough for sufficient inflation to occur, but still fast enough that the present day Universe would be in the true vacuum. 
In some theories the false vacuum is phenomenologically the desirable one. In many supersymmetric models, the desirable state actually turns out to be metastable, whereas the true ground state has undesirable properties such as spontaneous breaking of the QCD colour symmetry. Several models of supersymmetry breaking arising in a hidden sector and communicated to the observed sector by messengers, end up with unphysical ground states. In such cases one invokes the possibility that the parameters of the theory make the tunneling rate much smaller than the expansion rate of the universe till the present epoch. If a volume of the size $M^{-3}$ determined by the energy scale $M$ of the high energy theory is to not undergo a transition within the typical expansion time scale of the Universe, then

$$
\Gamma<M^{3} H_{0}
$$

where $H_{0}$ is the present value of the Hubble parameter, i.e., the experimentally observed Hubble constant.

As another application, in Standard Model, the Higgs boson has a self interaction potential of the type discussed above. The exact form of the potential, namely, which local minimum is energetically favored and what can be the tunneling rate for going from one vacuum to another is determined by the mass parameter and the quartic coupling occurring in the Higgs potential. The mass parameter is known from the requirement of spontaneous symmetry breaking to reproduce the Weak interaction scale. However, the quartic coupling will be determined only when the collider experiments will determine its mass. Since this value is as yet unknown, we can use cosmology to put a bound on its possible values.

It can be shown that if the Higgs boson is very light, then there is a danger for the Universe to be trapped in an unphysical vacuum. This puts a lower bound on the Higgs boson mass at about $10 \mathrm{GeV}$. This is known as the Linde-Weinberg bound.

\section{Inflationary Universe}

It is remarkable that the Friedmann-Robertson-Walker model is so successful a description of the observed Universe. At first this seems a resounding triumph of General Relativity. It is true that the dynamics all the way back to Big Bang Nucleosynthesis (BBN) is successfully described. However one begins to notice certain peculiarities of the initial conditions. First of all, the Big Bang itself presents a problem to classical physics, being a singularity of spacetime. But we expect this to be solved by a successful theory of Quantum Gravity. But now we will show that even the conditions existing after the Big Bang and well within the realm of classical General Relativity pose puzzles and demand a search for new dynamics or newer laws of physics.

\subsection{Fine tuned initial conditions}

Any physical entity such as a planet or a star or a galaxy has an associated legth or mass scale. Very often this is set by accidental initial conditions. However not all accidentally possible scales would be tolerated by the dynamics holding together the object. Some initial conditions will lead to unstable configurations. Question : what scales would be permitted by a universe driven according to General Relativity? The coupling constant of Gravity is dimensionful, $G_{N}^{-1}=M_{P l}^{2} \sim$ $\left(10^{19} \mathrm{GeV}\right)^{2}$. The available physical quantity is energy density. We propose a naive possibility that the system size or scale be decided by this energy density re-expressed in the units of $M_{P l}$. We then find that the Universe has far too small an energy density $10^{-6}(\mathrm{eV})^{4} \sim 10^{-66}\left(\mathrm{M}_{\mathrm{Pl}}\right)^{4}$, and far too big a size, Giga parsec compared to $10^{-20}$ fermi. It is interesting that Gravity permits a viable solution with such variance from its intrinsic scales.

Further, we find that above mentioned ratio evolves with time because the scale factor grows and density keeps reducing. We can ask what is the time scale set by gravity for this variation. In the evolution equation for the scale factor $R(t)$ we see that the intrinsic scale is set by the Gravitational constant. If the $\rho /\left(M_{P l}\right)^{4}$ was order unity at some epoch, then there is only one independent time scale left, that set by $M_{P l}$. We then find that the universe would either have 
recollapsed or expanded precisely within the time set by the Planck scale, $10^{-44}$ sec. The fact that the Universe seems to be hovering between collapse and rapid expansion even after 14 billion years, requires that we must start with extremely fine tuned initial conditions. The fine tuning has to be to the extent of one part in $10^{66}$ because we started with the value of the ratio close order unity.

It is such uncanny fine tuning that is the motivation for proposing an "inflationary" event in the early Universe, a phase of unusually rapid expansion. Such an event reconditions the ratios we discussed above. Their large apparant values then arise from dynamics rather than initial values. All physical realizations of this proposal have consisted of admitting dynamics other than Gravity to intervene for the purpose of significantly reconditioning these ratios.

It is fully likely that the answer to the puzzles to be described in the following is buried in the Planck era itself. Any data which can throw light on such a mechanism would also provide a valuable window into Planck scale physics. But there are viable candidates within the known physical principles of Relativistic Field Theory, a possibility which if true would reduce our intrinsic ignorance of the physical world, and in turn lead to prediction of newer forces.

\subsection{Horizon problem}

This problem arises from the fact that our Universe had a finite past rather than an indefinitely long past. A finite past gives rise, at any given time, to a definite physical size over which information could have travelled upto that time. This physical scale is called the "particle horizon". At present epoch we find unusually precise correlation in the physical conditions across many particle horizons, i.e., over regions of space that had no reason to be in causal contact with each other. It is to be noted that the world could well have emerged all highly correlated from the Planck era. But as explained above, we work in the spirit of exploring newer dynamics within the known principles.

With this preamble, the paradox presented by the current observations is as follows. We know that the observed Cosmic Microwave Background Radiation (CMBR) originated at the time of decoupling of photons from the partially ionized Hydrogen. The temperature of this decoupling, as we estimated in sec. 4.4 is $1200 \mathrm{~K}$, while today it is close to $1 \mathrm{~K}$. From the ratio of temperatures, and assuming a matter dominated Universe,

$$
\frac{T_{0}}{T_{d e c}} \simeq \frac{1}{1200}=\frac{R\left(t_{d e c}\right)}{R\left(t_{0}\right)}=\left(\frac{t_{d}}{t_{0}}\right)^{2 / 3}
$$

Therefore, $t_{d} \sim 2 \times 10^{5} h^{-1}$ years.

Now consider the size of the particle horizon at these two epochs, ie., the size of the region over which communication using light signals could have occurred since the Big Bang.

$$
a\left(t_{0}\right) \int_{t_{d}}^{t_{0}} \frac{d t^{\prime}}{R\left(t^{\prime}\right)} \approx 3 t_{0} \approx 6000 h^{-1} \mathrm{Mpc}
$$

where the contribution of the lower limit is ignorable. Similarly at $t_{d e c}$, horizon $\sim 3 t_{d e c} \approx$ $0.168 h^{-1} M p c$, using the time-temperature relation appropriate to the radiation dominated era. Then the angle subtended to us today by a causally connected region of the decoupling epoch is

$$
\theta_{d}=\frac{168}{6000} \simeq 0.03 \mathrm{rad} \text { or } 2 \mathrm{deg}
$$

This means that we are viewing today $\frac{4 \pi}{(0.03)^{2}} \approx 14,000$ causally unconnected horizon patches, and yet they show remarkable homogeneity.

\subsection{Oldness-Flatness problem}

An independent puzzle arises due to the fact that the curvature of the three dimensional space is allowed to be non-zero in General Relativity. Let us rewrite the evolution equation by dividing 
out by $H(t)^{2} \equiv(\dot{R}(t) / R(t))^{2}$

$$
\frac{1}{H(t)^{2}} \frac{k}{R(t)^{2}}=\Omega(t)-1
$$

where $\Omega(t) \equiv 8 \pi G \rho(t) / 3 H(t)^{2}$ which can be thought of as the energy density at time $t$ re-expressed in the units of $G$ and $H(t)$. At the present epoch $t_{0}$ in the Universe, the observational evidence suggests the right hand side (RHS) of above equation is \pm 0.02 . This suggests the simple possibility that the value of $k$ is actually zero. Let us first assume that it is non-zero and assume a power law expansion $R(t)=R_{0} t^{n}$, with $n<1$ as is true for radiation dominated and matter dominated cases. After dividing the previous equation on both sides by the corresponding quantities at present epoch $t_{0}$, we find

$$
\left(\frac{t}{t_{0}}\right)^{1-n}=50 \times(\Omega(t)-1)
$$

where we have used the current value of the right hand side (RHS), 0.02. Now the current value $t_{0}$ is $\approx 5 \times 10^{17}$ second, while at the time of Big Bang Nucleosynthesis (BBN) it was only about 100 seconds old. Using $n=1 / 2$ for the sake of argument, we find LHS $\approx 10^{-7}$, which means that correspondingly, on the RHS $\Omega$ must be tuned to unity to one part in $10^{8}$. If further, we compare to earlier epochs such as the QCD phase transition or the electroweak epoch, we need higher and higher fine tuning to achieve the 0.02 accuracy at present epoch. We thus see that the initial conditions have to be fine tuned so that we arrive at the Universe we see today. Equivalently, since the problem is connected to the large ratio of time scales on the LHS, we may wonder why the Universe has lived so long. This may be called the "oldness" problem.

In case the $k$ is zero, then that would be a miraculous fine tuning in itself. The discrete values $0, \pm 1$ arise only after scaling the curvature by a fiducial length-squared. The natural values for the curvature pass smoothly from negative to positive and zero is only a special point. The tuning of the value to zero earns this puzzle the name "flatness" problem.

We can also restate the problem as there being too much entropy in the present Universe. Taking the entropy density of the $\mathrm{CMB}$ radiation at $2.7 \mathrm{~K}$ and multiplying by the size of the horizon as set by approximately $H_{0}^{-1} \sim(1 / 3) t_{0} \sim 3 \times 10^{9}$ yr. we get the entropy to be $10^{86}$ ( check this!). The aim of inflationary cosmology is to explain this enormous entropy production as a result of an unusual phase transition in the early Universe.

\subsection{Density perturbations}

A last important question to be answered by Cosmology is the origin of the galaxies, in turn of life itself and ourselves. If the Universe was perfectly homogeneous and isotropic, no galaxies could form. Current observations of the distribution of several million galaxies and quasars suggests that the distribution of these inhomogeneities again shows a pattern. To understand the pattern one studies the perturbation in the density, $\delta \rho(\mathbf{x}, D)=\rho(\mathbf{x}, D)-\bar{\rho}$, where $\bar{\rho}$ is the average value and $\rho(\mathbf{x}, D)$ is the density determined in the neighborhood of point $\mathbf{x}$ by averaging over a region of size $D$. It is found that the density fluctuations do not depend on the scale of averaging $D$. It is a challenge for any proposal that purports to explain the extreme homogeneity and isotropy of the Universe to also explain the amplitude and distribution of these perturbations.

\subsection{Inflation}

The inflationary universe idea was proposed by A. Guth to address these issues by relying on the dynamics of a phase transition. The horizon problem can be addressed if there existed an epoch in the universe when the particle horizon was growing faster than the Hubble horizon. Later this phase ends and we return to radiation and matter dominated Universe.

If the Universe was purely radiation dominated during its entire early history particle horizon could not have grown faster than Hubble horizon. However, if there is an unusual equation of state obeyed by the source terms of Einstein's equations then this is possible. Our study of first order phase transitions suggests a possible scenario. We have seen that there is a possibility for 
the system ( the universe) to be trapped in a false vacuum. Exit from such a vacuum occurs by quantum tunneling. If this tunneling rate is very small, the vacuum energy of the false vacuum will dominate the energy density of the Universe. Vacuum energy of a scalar field has just the right property to ensure rapid growth of particle horizon, keeping the hubble horizon a constant. If inflation occurs, the flatness problem also gets automatically addressed.

Consider the energy momentum tensor of a real scalar field

$$
\begin{aligned}
T_{\nu}^{\mu} & =\partial_{\nu} \phi \partial^{\mu} \phi-\delta_{\nu}^{\mu} \mathcal{L} \\
& =\partial_{\mu} \phi \partial^{\mu} \phi-\delta_{\nu}^{\mu}\left(\frac{1}{2} \partial_{\lambda} \phi \partial^{\lambda} \phi-V(\phi)\right)
\end{aligned}
$$

If the field is trapped in a false vacuum, its expectation value is homogeneous over all space and is also constant in time. This means all the derivative terms vanish and $T_{\nu}^{\mu} \rightarrow V_{0} \delta_{\nu}^{\mu}$ where $V_{0}$ is the value of the potential $V$ in the false vacuum, called the vacuum energy density. Now for an isotropic and homogeneous fluid, the energy-momentum tensor assumes a special form, $\operatorname{diag}(\rho,-p,-p,-p)$. That is, the off diagonal terms are zero, the three space dimensions are equivalent, and the nonzero entries have the interpretation of being the usual quantities $\rho$ the energy density and $p$ the pressure. Thus comparing this form with that assumed by the scalar field in a false vacuum, we see that the expectation value of the scalar field behaves like a fluid obeying the unusual equation of state $p=-\rho$. Now the Friedmann equation becomes

$$
\left(\frac{\dot{R}}{R}\right)^{2}=\frac{8 \pi}{3} G \rho=\frac{8 \pi}{3} G V_{0}
$$

whose solution is $R(t)=R\left(t_{i}\right) \exp \left(H\left(t-t_{i}\right)\right)$, with $H^{2}=(8 \pi / 3) V_{0}$ and $t_{i}$ is some initial time.

\subsection{Resolution of Problems}

\subsubsection{Horizon and flatness problems}

During the inflationary epoch we assume an exponential expansion and a constant value of Hubble parameter $H$ (defined without special subscript or superscript since $H_{0}$ is reserved for the current value of $H$ ). Now consider the particle horizon, or equivalently, the luminosity distance at any epoch $t$

$$
d_{H}=e^{H t} \int_{t_{i}}^{t} \frac{d t^{\prime}}{e^{H t^{\prime}}} \approx \frac{1}{H} e^{H\left(t-t_{i}\right)}
$$

Thus the distance over which causal effects could be exchanged is exponentially larger than the simple estimate $3 t_{d e c}$ we used while discussing the horizon problem. Suppose inflation lasted for a duration $\left(t-t_{i}\right) \equiv \tau$. We can estimate what value $H \tau$ we need during inflation so that we are not seeing a large number of primordial horizon volumes but only about one. Suppose inflation ended leaving the Universe at a temperature $T_{r}$ ( subscript "r" signifies reheat as explained in next subsection). Then the $d_{H}$ of above equation rescaled to today assuming radiation dominated Universe 4 should give current inverse horizon $H_{0}^{-1}$. Thus

$$
\left(\frac{1}{H} e^{H \tau}\right) \times\left(\frac{T_{r}}{T_{0}}\right) \approx \frac{1}{H_{0}}
$$

In above formula let us estimate $H / H_{0}$ again assuming radiation dominated evolution since the $T_{r}$ till now,

$$
\left.\frac{H}{H_{0}} \sim \frac{t_{0}}{t}\right|_{F R W} \sim\left(\frac{T_{r}}{T_{0}}\right)^{2} \sim\left(\frac{10^{14} \mathrm{GeV}}{10^{-4} \mathrm{eV}}\right)^{2} \sim\left(10^{27}\right)^{2}
$$

\footnotetext{
${ }^{4}$ The Universe has of course not been radiation dominated through out. But replacing later history of the Universe by matter dominated evolution will not significantly alter these estimates since the expansion is changed by a small change in the power law, $t^{2 / 3}$ instead of $t^{1 / 2}$. Assumption of radiation dominated expansion allows relating temperatures at two different epochs, as a good approximation.
} 


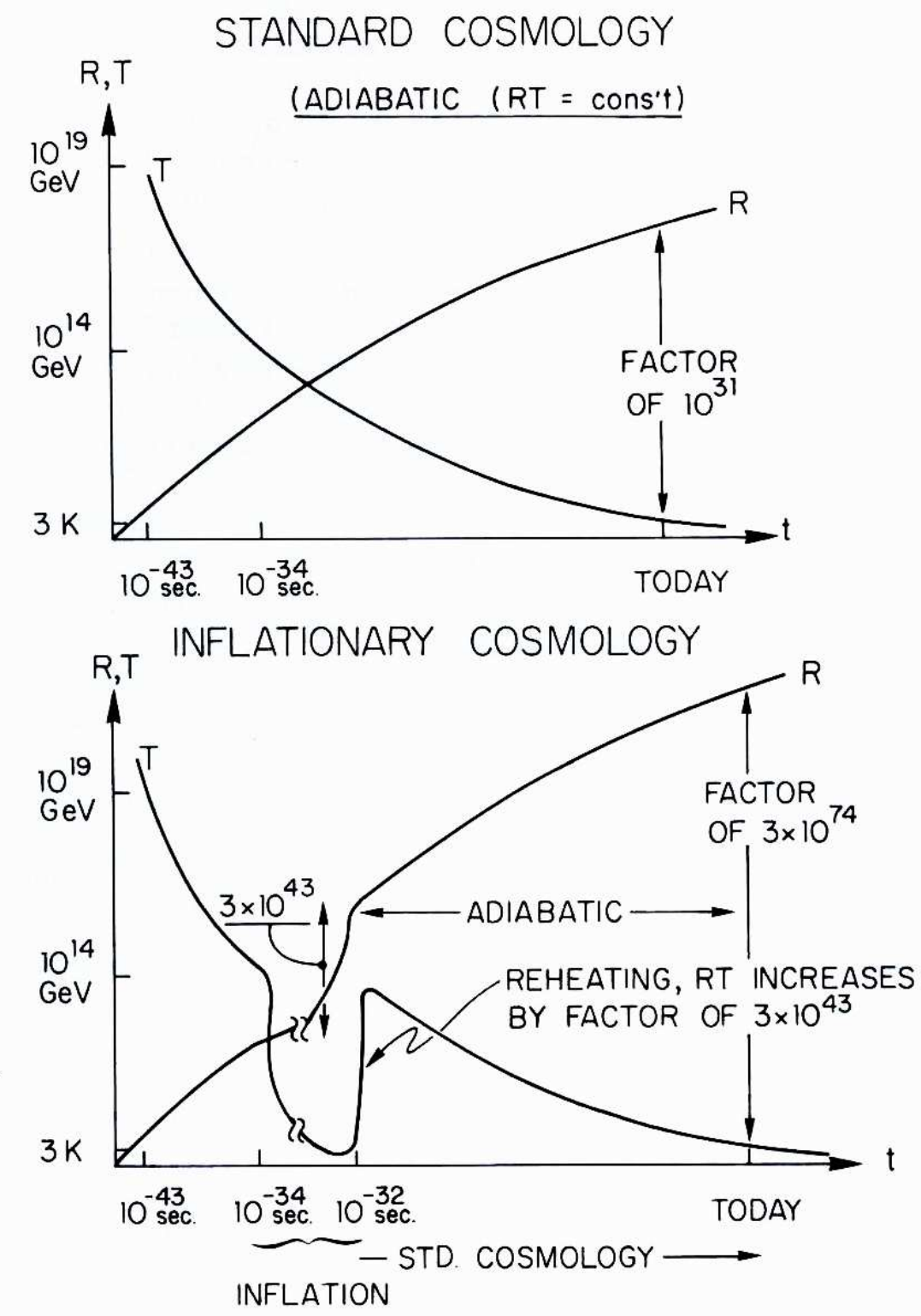

Figure 6: Comparison of simple FRW cosmology with inflationary cosmology. Figure courtesy Kolb and Turner. 
recall that $H^{2} \propto T^{4}$ and we inserted a Grand Unification scale $10^{14} \mathrm{GeV}$ as a possibility for $T_{r}$. Then $e^{H \tau}$ has to be $10^{27}$.

We need an improvement upon this estimate. As we shall later see, there are theoretical reasons to believe that $T_{r} \lesssim 10^{9} \mathrm{GeV}$. In this case it is also assumed that there is additional ( non-inflationary or mildly inflationary) stretching by a factor $10^{9}$. In this case we delink the reheat temperature $T_{r}$ from the vacuum energy density causing inflation, and assume the latter to continue to be at GUT scale. In this case,

$$
e^{H \tau} \sim\left(\frac{H}{H_{0}}\right) \times\left(\frac{t_{0}}{T_{r}}\right) \times 10^{-9} \sim 10^{18}
$$

where we have used estimate of the vacuum energy density $(0.03 \mathrm{eV})^{4}$ as derived from the directly observed value of current Hubble constant. In the literature one often sees this estimate made with GUT scale taken to be $10^{16} \mathrm{GeV}$ so that the required value of $e^{H \tau} \gtrsim 10^{22} \sim e^{55}$.

Let us see the requirement to solve the flatness problem. We need to show that the term $|k| / R^{2}$ becomes insignificant regardless of its value before inflation. For naturalness we assume it to be comparable to $H^{2}$ as expected from Friedmann equation. Now including scaling from inflation, the stretching by factor $10^{9}$ as introduced above, and subsequently during the era after reheating, we find

$$
\left(\frac{k}{R^{2}}\right)_{0} \sim\left(\frac{k}{R^{2}}\right)_{\text {pre-inf }} \times e^{-2 \tau} \times\left(10^{9}\right)^{-2}\left(\frac{T_{0}}{T_{r}}\right)^{2}
$$

The left hand side is $(1-\Omega) H_{0}^{2}$ which is insignificant. The right hand side is the same small factor we estimated above, squared. So this means the left hand side is reduced to a value $10^{-36}$ or $10^{-44}$ depending on the value of Grand Unification scale we take.

\subsubsection{Avoidance of unwanted relics}

A byproduct of Inflation is that it would also explain absence of exotic relics from the early Universe. Typically a grand unified theory permits occurance of topological defects such as cosmic strings or monopoles. They signifiy unusual local vacua of spontaneously broken gauge theories which cannot evolve by unitary quantum mechanical processes to the simple vacuum. Unlike normal heavy particle states, therefore, such defects cannot decay.

The natural abundance of such relics can be calculated by understanding the dynamics of their formation. Typically these events are the phase transitions characterised by specific tempratures. If the naturally suggested adundances of these objects really occured, they would quickly dominate the energy density of the Universe, with possible exception of cosmic strings. This would be completely contradictary to the observations. On the other hand if the scale of Inflation was below the temperature of such phase transitions, the density of topological objects formed would be diluted by the large factor by which volumes expand during inflation.

Another class of exotic relics are the so called moduli fields, scalar excitations arising in supersymmetric theories and String Theory. They are generic because of the powerful symmtry restrictions on potential energy functions in such theories. Inflation provides a solution for some class of models for this case also.

\subsubsection{Resolution for density perturbations}

Finally the density perturbations are neatly explained by inflation. The scalar field is assumed to be in a semi-classical state. However quantum fluctuations do exist and these should in principle be observable. Inflationary era is characterized by Hubble parameter $H$ remaining a constant while the scale factor grows exponentially. Thus the wavelengths of various Fourier components of the fluctuations are growing rapidly, leaving the horizon.

We shall take up in greater detail the theory of small perturbations in an expanding Universe in section 8 . There we show that the amplitudes of the Fourier modes of these perturbations 
remain frozen at the value with which they left the Hubble horizon. Eventually when the Universe becomes radiation dominated and subsequently matter dominated, Hubble horizon $H^{-1}$ begins to grow faster than the scale factor and the wavelengths of the modes begin to become smaller than Hubble horizon. This is the same reason as the solution of the horizon problem wherein apparently uncorrelated regions of distant space now seem to be correlated. They all emerged from the same causally connected region and got pushed out of the horizon during the inflation epoch.

The result of this evolution of the fluctuations is that when they re-enter the horizon they all have the same amplitude. These fluctuations then influence the rest of the radiation and matter causing fluctuations of similar magnitude in them. This is the explanation for the scale invariant matter density perturbations represented by distribution of galaxies. We shall take up the details of fluctuations in the next section.

\subsection{Inflaton Dynamics}

Inflation is a paradigm, a broad framework of expectations rather than a specific theory. The expectations can be shown to be fulfilled if a scalar field dubbed "inflaton" obeying appropriate properties exists. If inflation is implemented by such a scalar field, we need to make definite requirements on its evolution. We assume that its evolution leads the Universe through the following three phases

- Inflationary phase

- Coherent oscillation phase

- Decay and re-heating phase ("re-heat" only for low field scenario)

Of these three phases, the inflationary phase addresses the broadest requirements discussed in previous subsection. To obtain exponential expansion we need constant vacuum energy. This means that the field $p h i$ has a value where $V(\phi) \neq 0$ and also that $\phi$ continues to remain at such a value. The simplest such possibility is a false vacuum, a local minimum of the effective potential which is not a global minimum. But this is problematic because this kind of state can be far too stable and the exit from it keeping the Universe permanently inflating. Two possibilities which are strong candidates due to phenomenological reasons are the so called High Field (older name Chaotic Inflation) or the Low Field ( older name New Inflation) scenarios of inflation. These are shown in figure 7. In the High Field case the initial value of the field is close to Planck scale and no clear barrier separating it from the low energy true minimum. However its dynamics governed by Planck scale effects is "chaotic" and keeps it at a very high energy for a long time. In the Low Field case, the dynamics is usual field theory but the effective potential function has a long plateau of very small slope. Assuming the initial value of the field at the top of the plateau, this allows the field to sustain a position of large vacuum energy for a long time. In both cases, the field eventually moves towards the low energy true minimum, via the next two phases. A third possibility which is appealing for supersymmetric unified theories is called the Hybrid scenario. It involves two fields, one which keeps the Universe initially at a high field value, and the second field which becomes more dominant at a later stage, causing a rapid roll down and exit from the high energy plateau. It will not be possible for us to discuss these scenarios in any detail in these notes.

Next, it is easier to explain the third listed phase, since it is necessary that the end point of inflationary expansion is a hot Universe. Big Bang Nucleosynthesis is very successful in explaining the natural abundance of elements. We need to assume a hot Universe of at least a few $\mathrm{MeV}$ temperature for BBN to remain viable.

Finally, the intermediate phase dominated by coherent 5 oscillations of the scalar field is almost certain to occur as inflation ends. This is because during inflation the field is already in a coherent state, one in which it has homogeneous ( position independent) value. This phase ends with creation of quanta. In a large class of models this phase may be of no particular interest. But

\footnotetext{
${ }^{5}$ i.e., a special state in which it is possible to treat the field in the leading order as if it were a classical field.
} 

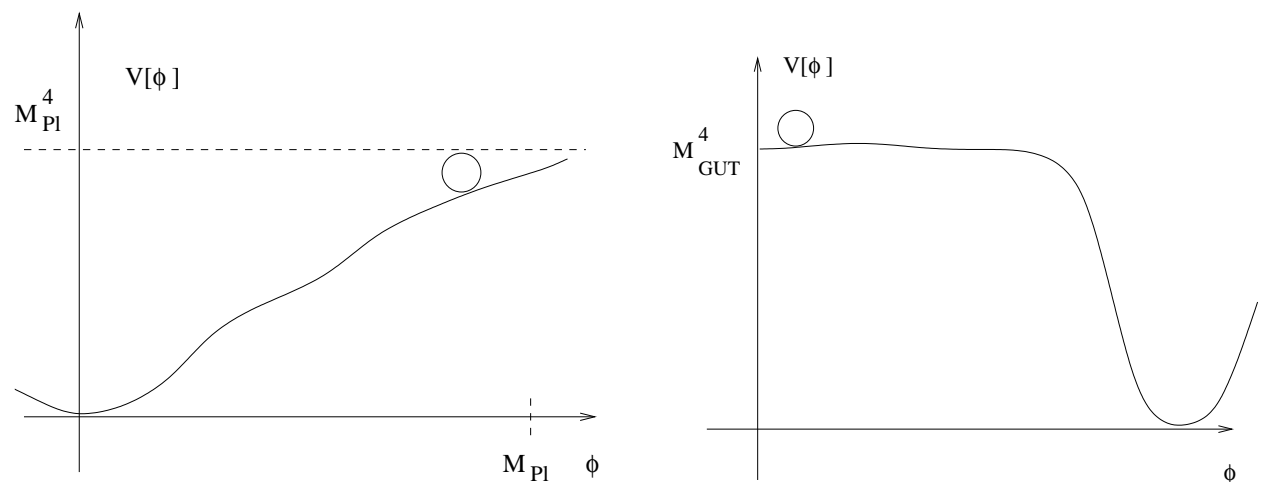

Figure 7: Sketches of stipulated effective potentials with the corresponding initial value of the inflaton field indicated by a small circle ( a "ball" ready to "roll down" the shown profiles). Left panel shows the High Field scenario, the right panel shows the Low Field scenario

in special cases when the coupling of the inflaton to other matter is tuned to certain values, it can lead to a variety of interesting effects which can have observable consequences. One such possibility is a long duration of coherent oscillations, which can be shown to mimic a Universe filled with pressureless dust. Alternatively there can be particles with special values of mass which can be shown to be produced copiously during the coherent oscillation epoch. Such effects are called "preheating" because heating of the Universe is achieved not directly through the inflaton but by other particles which have efficiently carried away the energy of the inflaton. Such phases can enhance the expansion achieved during the inflationary phase, the additional $10^{9}$ factor used in estimates in previous subsection.

\subsubsection{Predictions and observables}

For a quantitative study we consider a scalar field $\phi$ with lagrangian

$$
S[\phi]=\int d^{4} x \sqrt{-g}\left(\frac{1}{2} \partial_{\mu} \phi \partial^{\mu} \phi-V(\phi)+\mathcal{L}_{\phi-\text { matter }}\right)
$$

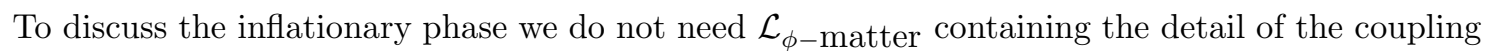
of the scalar field to the rest of matter. Varying this action after putting the Friedmann metric for $g_{\mu \nu}$ gives an equation of motion for $\phi$,

$$
\ddot{\phi}+3 H \dot{\phi}+V^{\prime}(\phi)=0
$$

The inflationary phase is characterized by a homogeneous value of $\phi$ and a very slow time evolution so that there is domination by vacuum energy. Mathematically one demands

$$
\ddot{\phi} \ll 3 H \dot{\phi}
$$

in other words we assume that the time scale of variation of the field $\phi$ encoded in $\dot{\phi} /{ }^{\cdot} \cdot \phi$ is small compared to the time scale $H^{-1}$ of the expansion of the Universe. Further, the assumption that the time scale of variation of $\phi$ is very small amounts to assuming that in the action we must have

$$
\dot{\phi}^{2} \ll V_{0}
$$

Thus while obtaining the Euler-Lagrange equations the $V$ term continues to be important, but the higher time derivative of $\phi$ can be dropped. So the evolution equation reduces to

$$
3 H \dot{\phi}=-V^{\prime}(\phi)
$$


To ensure consistency of the above two conditions, divide the simplified evolution equation by $3 H$ and take a time derivative. Recall that $H^{2}=(8 \pi / 3) G V(\phi)$. Thus $H$ can be implicitly differentiated with respect to time as shown in the equation below

$$
\ddot{\phi}=-\frac{V^{\prime \prime}(\phi) \dot{\phi}}{3 H(\phi)}+\frac{V^{\prime}(\phi)}{3 H^{2}(\phi)} H^{\prime}(\phi) \dot{\phi}
$$

We now write the equation in three equivalent forms

$$
\begin{aligned}
\frac{\ddot{\phi}}{3 H \dot{\phi}} & =-\frac{V^{\prime \prime}(\phi)}{9 H^{2}}+\frac{V^{\prime}}{9 H^{3}} H^{\prime} \\
& =-\frac{V^{\prime \prime}(\phi)}{9 \times \frac{8 \pi}{3} G V_{0}}+\frac{V^{\prime}\left(\frac{4 \pi}{3}\right) G V^{\prime}}{9 \times\left(\frac{8 \pi}{3} G\right)^{2} V^{2}} \\
& =-\frac{V^{\prime \prime}}{3 \times 8 \pi G V_{0}}+\frac{1}{2} \frac{1}{3 \times 8 \pi G}\left(\frac{V^{\prime}}{V}\right)^{2}
\end{aligned}
$$

where use has been made of the preceding assumptions as also the relation $2 H H^{\prime}=\frac{8 \pi}{3} G V^{\prime}$.

Inspecting the above equations we define three parameters

$$
\epsilon \equiv \frac{M p^{2}}{16 \pi}\left(\frac{V^{\prime}(\phi)}{V(\phi)}\right)^{2}, \quad \eta \equiv \frac{M p^{2}}{8 \pi} \frac{V^{\prime \prime}(\phi)}{V(\phi)}
$$

and

$$
\xi \equiv \frac{\ddot{\phi}}{H \dot{\phi}}=\epsilon+\eta
$$

The requirements of the inflationary phase viz., large vacuum energy and a vary slow roll towards the true minimum mean that

$$
\epsilon \ll 1,|\eta| \ll 1 \text { and } \xi \sim 0(\epsilon, \eta)
$$

We use these in the criterion that if either of these quantities becomes large, inflationary phase ends. These have come to be called the "slow roll" parameters characterizing inflation.

For several decades inflation remained a theoretical paradigm. However with precision cosmological experiments such as the Hubble Space Telescope (HST) and the Wilkinson Microwave Anisotropy Probe (WMAP) yielding valuable data we face the exciting prospects of verifying and refining the paradigm, and also deducing the details of the dynamics of the inflaton field. It is possible to set up a relationship between slow roll parameters introduced above and the temperature fluctuation data of the microwave background radiation. Similarly the large scale galaxy surveys such as 2dF GRS ( 2 degree Field Galaxy Redshift Survey) and 6dF GRS provide detailed data on distribution of galaxies which can be counter checked over a certain range of wavenumbers against the fluctuations as observed in WMAP and also against the specific dynamics of the inflaton.

Finally let us work out a simple example of how we can relate intrinsic properties of the effective potential to observable features of inflation. We can for instance compute the number of e-foldings $N$ in the course of $\phi$ evolving from initial value $\phi_{i}$ to a value $\phi_{f}$, given the form of the potential.

$$
\begin{aligned}
N\left(\phi_{i} \rightarrow \phi_{f}\right) & \equiv \ln \left(\frac{R\left(t_{f}\right)}{R\left(t_{i}\right)}\right)=\int_{t_{i}}^{t_{f}} H d t=\int \frac{H}{\dot{\phi}} d \phi \\
& =-\int_{\phi_{i}}^{\phi_{f}} \frac{3 H^{2} d \phi}{V^{\prime}}
\end{aligned}
$$


where we assumed $t_{f} \sim H^{-1}\left(\phi_{f}\right)$. Now shift the global minimum of the effective potential to be at $\phi=0$ so $\phi_{i} \gg \phi_{f}$

$$
=-8 \pi G \int_{\phi_{i}}^{\phi_{f}} \frac{V(\phi)}{V^{\prime}(\phi)} d \phi
$$

This formula can be used to relate the dominant power law in the effective potential with the number of e-foldings. For $V(\phi)=\lambda \phi^{\mu}$,

$$
\begin{aligned}
N\left(\phi_{i} \rightarrow \phi_{f}\right) & =\frac{4 \pi}{\nu} G\left(\phi_{0}^{2}-\phi_{f}^{2}\right) \\
& \approx\left(\frac{4 \pi}{\nu} G\right) \phi_{i n t}^{2}
\end{aligned}
$$

\section{Density perturbations and galaxy formation}

An outstanding problem facing FRW cosmological models is formation of galaxies. If the Universe emerged from the Planck era perfectly homogeneous and isotropic, how did the primordial clumping of neutral Hydrogen occur? Without such clumping formation of galaxies and in turn stars would be impossible.

The related observational facts are also challenging. The fluctuations in the average density have resulted in a distribution of galaxies and clusters of galaxies. What is remarkable is that these fluctuations exist at all observable scales. Further, the observed fluctuations seem to have originated from seed fluctuations which were of the same magnitude, approximately one part in $10^{5}$, independent of the scale at which we study the fluctuations. This statement of scale invariance began as a hypothesis, known as the Harrison-Zel'dovich spectrum but has been remarkably close to the extensive experimental evidence accumulated over the last fifty years. The main sources of current data are Sloan Digital Sky Survey (SDSS), Two degree field Galaxy Redshift Survey (2dF GRS), quasar redshift surveys, "Lyman alpha forest" data etc, collectively called Large Scale Structure (LSS) data.

Here we shall present a brief overview of the formalism used for studying fluctuations. We shall also show that the inflationary Universe is in principle a solution, providing scale invariant fluctuations. The magnitude of resulting fluctuations however is too large unless we fine tune a parameter to required value.

\subsection{Jeans Analysis for Adiabatic Perturbations}

First we study the evolution of perturbations in a non-relativistic fluid. We study the continuity equation, the force equation and the equation for gravitational potential assuming that the state of the fluid provides a solution a solution to these. We then work out the equations satisfied by the perturbations. Fourier analysing the perturbations, it is found that modes with wavelengths larger than a critical value of the wavelength the perturbations are not stable.

The relevant equations for the mass density $\rho(\mathbf{x}, t)$, velocity field $\mathbf{v}(\mathbf{x}, t)$ and the newtonian gravitational potential $\phi(\mathbf{x}, t)$ are

$$
\begin{aligned}
\frac{\partial \rho}{\partial t}+\nabla \cdot(\rho \mathbf{v}) & =0 \\
\frac{\partial \mathbf{v}}{\partial t}+(\mathbf{v} \cdot \nabla) \mathbf{v}+\frac{1}{\rho} \nabla p+\nabla \phi & =0 \\
\nabla^{2} \phi & =4 \pi G \rho
\end{aligned}
$$


We now split the quantities into average values $\bar{\rho}, \bar{p}$ and space-time dependent perturbations $\rho_{1}$, $p_{1}$

$$
\rho(\mathbf{x}, t)=\bar{\rho}+\rho_{1}(\mathbf{x}, t) \quad p(\mathbf{x}, t)=\bar{p}+p_{1}(\mathbf{x}, t)
$$

and similarly for velocity. However, for a homogeneous fluid, average velocity is zero so $\mathbf{v}$ and $\mathbf{v}_{1}$ are the same. It is reasonable to assume that there is no spatial variation in equation of state. This means the speed of sound is given by

$$
v_{s}^{2}=\left(\frac{\partial p}{\partial \rho}\right)_{\text {adiabatic }}=\frac{p_{1}}{\rho_{1}}
$$

Thus the equations satisfied by the fluctuations are,

$$
\begin{aligned}
\frac{\partial \rho_{1}}{\partial t}+\bar{\rho} \nabla \cdot \mathbf{v}_{1} & =0 \\
\frac{\partial \mathbf{v}_{1}}{\partial t}+\frac{v_{s}^{2}}{\bar{\rho}} \nabla \rho_{1}+\nabla \phi_{1} & =0 \\
\nabla^{2} \phi_{1} & =4 \pi G \rho_{1}
\end{aligned}
$$

From these coupled equations we obtain a wave equation for $\rho_{1}$,

$$
\frac{\partial^{2} \rho_{1}}{\partial t^{2}}-v_{s}^{2} \nabla^{2} \rho_{1}=4 \pi G \bar{\rho} \rho_{1}
$$

whose solution is

$$
\begin{aligned}
\rho_{1}(\mathbf{r}, t) & =A e^{(i \omega t-i \mathbf{k} \cdot \mathbf{r})} \\
\text { with } \omega^{2} & =v_{s}^{2} k^{2}-4 \pi G \bar{\rho}
\end{aligned}
$$

The expression for $\omega$ suggests the definition of a critical wavenumber, Jeans wavenumber, $k_{J} \equiv$ $\left(4 \pi G \bar{\rho} / v_{s}^{2}\right)^{1 / 2}$. For $k \ll k_{J}$ we get exponential growth, i.e., instability. We can understand this result by associating with a wavelength $\lambda$ a hydrodynamic timescale $\tau_{\text {hyd }} \sim \lambda / v_{s} \sim 1 / k v_{s}$. This is the timescale during which pressure differences will be communicated by perturbations with wavelength $\lambda$. Next we associate the timescale $\tau_{\text {grav }}=\left(4 \pi G \rho_{o}\right)^{-1 / 2}$ with the gravitational influences. The result above says that for the wavelengths for which hydrodynamic response is slower to propagate than gravitational influences, the latter win and cause a gravitational collapse.

We can now define Jeans mass

$$
M_{J} \equiv \frac{4 \pi}{3}\left(\frac{\pi}{k_{J}}\right)^{3} \rho_{o}=\frac{\pi^{5 / 2}}{6} \frac{v_{s}{ }^{3}}{G^{3 / 2} \rho_{0}^{1 / 2}}
$$

We can deduce that a homogeneous mass bigger than this value is susceptible to gravitational collapse.

\subsection{Jeans Analysis in Expanding Universe}

We need to extend the above treatment to the case of expanding Universe. Firstly we must let the mean density and pressure be time dependent due to change in cosmological scale factor. For instance, the mean density in a matter dominated universe will scale as $\bar{\rho}(t)=\bar{\rho}\left(t_{i}\right)\left(R\left(t_{i} / R(t)\right)^{3}\right.$ where $t_{i}$ denotes some "initial" reference time.

From now on we shall not follow the evolution of the other quantities but focus on the energy density. We introduce the dimensionless quantity $\delta=\rho_{1} / \bar{\rho}$ from which the obvious $R(t)$ dependence gets scaled out. Further, using the FRW metric in the comoving form, we treat $\mathbf{r}$ to be 
dimensionless and $t$ and $R(t)$ to have dimensions of length ( equivalently, time). Introduce the Fourier transform

$$
\delta(\mathbf{x}, t)=\int \frac{d^{3} k}{(2 \pi)^{3}} \delta_{\mathbf{k}}(t) e^{(-i \mathbf{k} \cdot \mathbf{r})}
$$

It can be shown that these Fourier modes obey the equation

$$
\ddot{\delta}_{k}+\frac{2 \dot{R}}{R} \dot{\delta}_{k}+\left(\frac{v_{s}^{2} k^{2}}{R^{2}}-4 \pi G \bar{\rho}(t)\right) \delta_{k}=0
$$

We see that the modified Jeans wave number defined by

$$
k_{J}^{2} \equiv 4 \pi G \bar{\rho}(t) R(t)^{2} / v_{s}^{2}
$$

plays a crucial role, in that at any given epoch $t$, wavelengths shorter than $\sim 1 / k_{J}$ are oscillatory and hence stable.

We shall now study the fate of the long wavelengths, the ones significant on cosmological scales. We shall see that the unstable modes grow in time, but instead of exponential growth they can have power law growth. We have assumed $k \rightarrow 0$. Further, using FRW equation we can replace the $G \bar{\rho}(t)$ term by $(3 / 2)(\dot{R} / R)^{2}$ in a spatially flat universe. Then for matter dominated universe with $R \propto t^{2 / 3}$, we get

$$
\ddot{\delta}+\frac{4}{3 t} \dot{\delta}-\frac{2}{3 t^{2}} \delta=0
$$

The solutions are

$$
\begin{aligned}
& \delta_{+}(t)=\delta_{o}\left(t_{i}\right)\left(\frac{t}{t_{i}}\right)^{2 / 3} \\
& \delta_{-}(t)=\delta_{-}\left(t_{i}\right)\left(\frac{t}{t_{i}}\right)^{-1}
\end{aligned}
$$

Studying the examples of other power law expansions of scale factor $R$, one may conclude that the expansion of the universe keeps pulling apart the infalling matter and slows down the growth of Jeans instability. However In the case of de Sitter universe it is found that exponential instability persists. In this case the long wavelength modes obey the equation

$$
\ddot{\delta}+2 H \dot{\delta}-\frac{3}{2} H^{2} \delta=0
$$

so that substituting $\delta \sim e^{\alpha t}$ we find

$$
\alpha^{2}+2 H \alpha-\frac{3}{2} H^{2}=0
$$

This has the roots $\alpha_{ \pm}=-H \pm \sqrt{H^{2}+3 / 2}$. One root is negative definite signifying decaying exponential though one positive root persists, $\alpha_{+}=\sqrt{H^{2}+3 / 2}-H$.

\subsubsection{Fate of the super-horizon modes}

Let us now return to the idea of inflationary universe as the source of primordial perturbations. The basic hypothesis is that the quantum mechanics of the "inflation" scalar field causes fluctuations in its expectation value. These then manifest as perturbations in the classical quantity, the energy density. This assumption is expressed as

$$
\rho_{1} \equiv \delta \rho=\frac{\delta V}{\delta \phi} \delta \phi
$$

where

$$
\delta \phi \equiv\left\langle(\phi-\langle\phi\rangle)^{2}\right\rangle
$$


and the expectation values are computed in an appropriately chosen state. This choice is not always easy. In general this is a static, translation invariant state with similar properties shared by the vaccum expectation values. In an expanding Universe the corresponding symmetries available are those of the space-time metric, namely the FRW metric. Since the time translation symmetry is lost, there are several conceptual issues. Fortunately the de Sitter metric has a sufficiently large group of symmetries permitting a fairly unique choice of the vaccum. Inflationary universe resembles the de Sitter solution over a substantial length of time so that we can adopt the answers obtained for the de Sitter case.

Decompose $\delta \phi$ into Fourier modes with the same conventions as in the preceding section. During the inflationary phase, the expectation value of $\phi$ remains approximately constant. Hence by appropriate shifting of the field, dynamics of $\delta \phi$ and $\phi$ are the same. The equations of motion for the modes of $\delta \phi$ are then

$$
\delta \ddot{\phi}_{k}+3 H \delta \dot{\phi}_{k}+k^{2} \frac{\delta \phi_{k}}{R^{2}}=0
$$

Then for "super-horizon" fluctuations with wavenumbers satisfying $k \ll R H$, we can ignore the last term and the non-decaying solution is $\delta \phi_{k}=$ constant. This is a crude argument to justify that the fluctuations at this scale become constant in amplitude. But constant amplitude would mean vanishing time derivatives, so the third term can't be smaller than the first two. In order to consistently ignore the third term relative to the second, we need to additionally assume that if the time scale of variation of $\delta \phi_{k}$ is $\tau$, then $1 / \tau>k / R$ in addition to $k \ll R H$. The two inequalities together imply $1 / \tau \gg H$. Thus the fluctuations are constant in the sense that the time scale of their variation is much less than the natural time scale of the geometric background, $H^{-1}$.

The above statement can be made more precise using Quantum Field Theory in curved spacetime, where it can be shown that in de Sitter universe, for a massless scalar field, the fluctuations in $\phi$, after appropriate cut-off procedure, are given by

$$
\left\langle\phi^{2}\right\rangle=\left(\frac{H}{2 \pi}\right)^{2}
$$

The assumption in this calculation is that the state chosen is de Sitter invariant. Thus variations that do not respect this invariance don't constribute and we get the expected constant result. Further, since no new scales are introduced through the choice of the state, the only dimensionful quantity available in the problem is $H$, which effectively determines the magnitude of the fluctuations.

We can now see the qualitative features which make inflation so appealing for generating scale invariant perturbations. For all normal kind of states of matter and energy, the scale factor grows as power law $t^{s}$, with $s<1$. Thus $H^{-1}$ grows as $t$ and all wavelengths $\lambda_{p h y}$ scale as $\left(\lambda_{p h y} / R\left(t_{1}\right)\right) \times$ $R(t)$ and hence keep falling inside the horizon. On the other hand, in the inflationary phase, $H^{-1}$ $=$ constant and wavelengths grow exponentially, $\lambda_{p h y}(t) \propto \lambda_{i} e^{H\left(t-t_{i}\right)}$ with some reference initial time $t_{i}$. Later, after inflation ends, $H^{-1}$ begins to grow faster than other length scales and steadily catches up with fluctuations of increasing values of wavelengths. Between leaving $H^{-1}$ and reentering $H(t)^{-1}$ later, the amplitude of the fluctuations remains frozen by arguments of preceding para. Note that for other power law expansions $t^{s}$ with $s>1$ also, the wavelengths grow faster than the Hubble horizon. However, the amplitude of these wavelengths will not remain constant and will not reproduce the scale invariant spectrum after re-entering the horizon. Some of these arguments will become clearer in the following subsection.

\subsubsection{Connection to density and temperature perturbations}

The problem of galaxy formation is to predict the observed pattern of clumping of luminous matter. Also, since matter and radiation were in equilibrium upto decoupling, the fluctuations in matter have also to be reflected in the fluctuations in the temperature of the CMBR.

The fluctuation we are referring to are in the spatial distribution. These are mathematically characterized by the auto-correlation function

$$
(\Delta \rho)^{2}(\mathbf{r}) \equiv\langle\delta \rho(\mathbf{x}) \delta \rho(\mathbf{x}+\mathbf{r})\rangle
$$


where on the right hand side, an averaging process is understood. For a homogeneous medium, the locations $\mathbf{x}$ are all equivalent and this dependence drops out at the end of averaging process. Introducing the Fourier transform $\delta_{\mathbf{k}}$, we can show that

$$
(\Delta \rho)^{2}(\mathbf{r})=\int d k \frac{k^{2}}{2 \pi^{2}}\left|\delta_{\mathbf{k}}\right|^{2} \frac{\sin k r}{k r}
$$

Now the rms value $\Delta \rho_{\text {rms }}$ at a given point is the square-root of this auto-correlation function for $r=0$. Accordingly, taking the limit $k r \rightarrow 0$ in the above expression, we get

$$
\left(\Delta \rho_{r m s}\right)^{2}=\int \frac{d k}{k} \mathcal{P}(k)
$$

with

$$
\mathcal{P}(k) \equiv \frac{k^{3}}{2 \pi^{2}}\left|\delta_{\mathbf{k}}\right|^{2}
$$

$\mathcal{P}$ represents the variation in $\left(\Delta \rho_{r m s}\right)^{2}$ with variation in $\ln k$. The aim of experiments is to determine the quantity $\mathcal{P}$.

Linear perturbation theory used above is valid only for small fluctuations. Once a fluctuation grows in magnitude it begins to be controlled by non-linear effects. We can estimate the intrinsic scale upto which the mass of a typical galaxy could have been in the linear regime. Using the value of the $\bar{\rho}$ to be the present abundance of non-relativistic matter ( $\approx 10^{-29} \mathrm{~g} / \mathrm{cc}$ ) and bringing out a factor of $10^{11}$ solar masses, the mass in a sphere of diameter of wavelength $\lambda$ is

$$
M \simeq 1.5 \times 10^{11} M_{\odot}\left(\Omega_{0} h^{2}\right)\left(\frac{\lambda}{\mathrm{Mpc}}\right)^{3}
$$

Assuming $10^{12}$ solar masses per galaxy, this gives the size $\lambda$ to be $1.9 \mathrm{Mpc}$, far greater than the actual galactic size $30 \mathrm{kpc}$. The $\lambda$ found here represents the size this mass perturbation would have had today had it not entered the non-linear regime.

Present data are not adequate to determine the spectrum $\mathcal{P}$ over all scales. However too large a magnitude of fluctuations at horizon scale would have been imprited on temparature fluctuations of CMB, which it is not. Likewise large fluctuations at smaller scales could have seeded gravitational collapse and given rise to a large number of primordial black holes, which also does not seem to be the case. Hence the spectrum must not be varying too greatly over the entire range of wavenumbers. It is customary to assume the spectrum of $\left|\delta_{\mathbf{k}}\right|^{2}$ to not involve any special scale, which means it must be a power law $k^{n}$. Further, a fluctuation of physical scale $\lambda$ contains mass $M \sim \lambda^{3} \sim k^{-3}$. Hence the spectrum $\mathcal{P} \sim M^{-1-n / 3}$. Now if we make the hypothesis that the spectrum of perturbations seems to be independent of the scale at which we observe it, we expect $\mathcal{P} \sim$ a constant, i.e. independent of $M$. For this to be true, $n$ must be -3 .

In the analyses of WMAP data of CMB it is customary to normalize the rms perturbation spectrum by its observed value at $(8 / h) \sim 11 \mathrm{Mpc}$ and denote it $\sigma_{8}$. WMAP reports the best fit value to be 0.8. Further, $\left(\Delta \rho_{r m s}\right)^{2}$ is parameterized as $k^{\left(-1+n_{s}\right)}$ where the subscript in $n_{s}$ signifies scalar perturbations. A very long epoch of perfectly de Sitter inflation would produce $n_{s}=1$ and a perfectly scale invariant spectrum. Given a specific model of inflation the small departures of $n_{s}$ from unity can be calculated as a function of $k$. This mild dependence of $n_{s}$ on $k$ is referred to as "running of the index" of the power law. Current WMAP data ( ie horizon scale perturbations) seem to suggest $n_{s}=0.97$ but the direct observations of LSS data ( galaxies and clusters of galaxies) suggest $n_{s}>1$.

Likewise a formalism exists for relating the temperature fluctuations with the density perturbations and in turn with the scalar field fluctuations. We shall not go into it here and the reader is referred to the references.

\subsection{Density Fluctuations from Inflation}

We now show how the inflation paradigm along with the knowledge of the form of the scalar potential helps us determine the magnitude of the scalar field fluctuations. 
Suppose we wish to know the fluctuation in a scale of size of our present horizon. According to the derivation in previous subsection we need to know the value of the perturbation when it left the horizon in the inflationary era. And we need to know the number of e-foldings the inflationary universe went through before becoming radiation dominated. It is the latter fact which then determines the later epoch when the same scale re-enters the horizon.

Let us trace a physical scale $\ell_{0}$ today by keeping track of corresponding co-moving value $\ell$. We have to consider the evolution in two parts. From the present we can only extrapolate back to the time when the current hot phase of the Universe began, i.e. the "re" heated phase. Prior to that was the phase of inflaton oscillation and decay. Reheating is assumed to be complete at a time $t_{d}$, the decay lifetime of the inflaton or its product particles.

Thus the size of a scale $\ell$ can be extrapolated to the epoch $t_{f}$ when inflation ended, (i.e., the slowness conditions on the evolution of the scalar field ceased to be valid) by

$$
\ell_{f}=\ell\left(\frac{T_{o}}{T_{r}}\right)\left(\frac{R\left(t_{f}\right)}{R\left(t_{d}\right)}\right)
$$

The last ratio can be estimated if we are given the effective potential $V(\phi)=\lambda \phi^{\nu}$ and a formalism for the dissipation of the inflaton vacuum energy. We shall not pursue these details here but claim that this can be calculated to be

$$
\begin{aligned}
\frac{R\left(t_{f}\right)}{R\left(t_{d}\right)} & =\left(\frac{t_{f}}{t_{d}}\right)^{(\nu+2) / 3 \nu} \\
& =\left(\frac{t_{f}}{t_{d}}\right)^{1 / 2} \quad \text { for } \nu=4
\end{aligned}
$$

Now $\ell_{\text {phys }}\left(t_{f}\right)=H^{-1} e^{N_{\ell}}$ where $N_{\ell}$ is the number of e-foldings between the time the specific scale attained the value $H^{-1}$, (i.e. became comparable to the horizon) and the end of inflation. Substituting the current value of the horizon in the above expressions finally gives $N_{H_{0}^{-1}} \approx 50-60$.

We now trace the magnitude of the perturbation through this exit from horizon followed by the re-entry at present epoch. It turns out that $\delta \rho / \rho$ is a physically ambiguous quantity to follow through such an evolution. This is because choice of a particular time coordinate amounts a choice of a gauge in General Relativity. The gauge invariant quantity to focus on has been shown to be $\zeta=\delta \rho /(p+\rho)$.

We seek the value of the numerator at a late epoch when inflation has ended. The denominator at this epoch is determined by $p=0$ and the energy density which is dominated by the kinetic term. The value of $\zeta$ at the inflationary epoch is known from preceding arguments about perturbations on scales comparable to horizon. Here

$$
\delta \rho=\frac{\delta V}{\delta \phi} \delta \phi=V^{\prime}(\phi) \frac{H}{2 \pi}
$$

where the $\delta \phi$ is estimated from QFT calculation of the rms value. Further, we replace $\dot{\phi}^{2}$ by using the slow roll condition of inflation, $3 H \dot{\phi}=-V^{\prime}(\phi)$. Thus we equate

$$
\begin{aligned}
\left(\frac{\delta \rho}{\dot{\phi}^{2}}\right)_{\ell \sim H^{-1}} & =\left.\zeta\right|_{\ell \sim H^{-1}} \\
& =\left.\frac{V^{\prime}(\phi) H(\phi)}{2 \pi \dot{\phi}^{2}}\right|_{\ell \sim H^{-1}} \\
& =\left(\frac{9 H^{3}(\phi)}{2 \pi\left(V^{\prime}(\phi)\right)}\right)_{\ell \sim H^{-1}}
\end{aligned}
$$

\footnotetext{
${ }^{6}$ We remind the reader that it is possible in some inflationary scenarios for the Universe to never have been in thermal equilibrium before this stage. Hence the prefix "re" is purely conjectural though conventional.
} 
Thus in matter dominated era when $p=0$, we have recovered

$$
\left(\frac{\delta \rho}{\rho}\right)_{\ell}=\left(\frac{2}{5}\right) \times 8 \sqrt{6 \pi} \frac{V^{3 / 2}\left(\phi_{\ell}\right)}{M_{P}^{2} V^{\prime}\left(\phi_{\ell}\right)}
$$

where we reexpress $G \equiv 1 / M_{P}^{2}$, the squared inverse of the Planck mass in natural units. The $2 / 5$ factor is acquired during transition from radiation dominated to matter dominated era.

We thus need the values of $V$ and $V^{\prime}$ at the value $\phi_{\ell}$. We do not really know the latter directly. But we can determine it if we know the number of e-foldings between its crossing the horizon and the end of inflation. Inverting the relation

$$
H^{-1}\left(\phi_{\ell}\right)=\ell_{p h y}\left(t_{f}\right) e^{N_{\ell}}
$$

for $\phi_{\ell}$ and also using

$$
N_{\ell}\left(\phi_{\ell} \rightarrow \phi_{f}\right)=\int H d t=\int_{\phi_{\ell}}^{\phi_{f}} \frac{H}{\dot{\phi}} d \phi \rightarrow \pi G \phi_{\ell}^{2}
$$

where the last arrow gives the answer corresponding to the form $\lambda \phi^{4}$ for the effective potential and is obtained by consistently using the slowness condition and the FRW equation. This gives us the number of e-foldings between horizon crossing by this scale and the end of inflation. Therefore, trading $N_{\ell}$ for $\phi_{\ell}$ we get

$$
\left(\frac{\delta \rho}{\rho}\right)_{\ell}=\frac{4 \sqrt{6 \pi}}{5} \lambda^{1 / 2}\left(\frac{\phi_{\ell}}{M_{P}}\right)^{3}=\frac{4}{5} \sqrt{6 \pi} \lambda^{1 / 2}\left(\frac{N_{\ell}}{\pi}\right)^{3 / 2}
$$

We have arrived at a remarkable mathematical relationship, expressing the magnitude of perturbations visible in the sky today with the parameters of the effective potential that drove the primordial inflation. We can take the fluctuations $\delta \rho / \rho$ to be as visible in the CMB temperature fluctuations, $\delta T / T \sim 6 \times 10^{-5}$. Assuming $N=55$ as needed for solving the horizon and flatness problems, we find that we need the value of $\lambda \sim 6 \times 10^{14}$. This is a tremendous theoretical achievement. Unfortunately the required numerical value is unnaturally small and it appears that we have to trade the fine tuning required to explain the state of the Universe with a fine tuning of a microscopic effective potential.

\section{Relics of the Big Bang}

As the Universe cools reaction rates of various physical processes become slow. When they become slower than the expansion rate of the Universe, the entities governed by those reactions no longer interact and remain as residual relics. The mathematical description for these events is provided by Boltzmann equations which can be used to infer the relative abundance of these relic particles which can be in principle observed today. The term relic applies to a wide variety of objects, including extended objects like cosmic strings or domain walls but we shall be dealing only with particle like relics in these notes.

\subsection{Boltzmann Equations}

Boltzmann equations describe the approach to equilibrium of a system that is close to equilibrium. In the context of the early Universe we have two reasons for departure from equilibrium. One is that if the reheat temperature after inflation has been $T_{\text {reh }}$ then all processes requiring energies larger than $T$ are suppressed and such processes play no role in establishing dynamical equilibrium. Thus particles that interact via only such processes remain out of equilibrium. We do not have much more control on such entities and in any case they most likely got inflated away and will not be recreated due to insufficient energy for them to be created.

The more important source of departure from equilibrium is the fact that the Universe is expanding. Like in an expanding gas, the temperature systematically falls. The primary assumption 
here is adiabaticity - i.e. extreme slowness of the rate of change of temperature compared to the time scales of the equilibrating processes. However, interesting epochs in the Universe correspond to times when the rates of a few specific processes are becoming as small as the expansion rate of the Universe. After the epoch is passed same reactions go out of equilibrium and the last conditions remain impritned as initial conditions for the rest of the evolution.

Schematically one can think of the Boltzmann equation as Liouville operator $\hat{L}$ acting on distribution function $f$, with a driving force provided by a collision operator $C$. In the absence of the collision term we have equilibrium statistical mechanics. $\hat{L}[f]=C[f]$ The Liouville operator which basically describes convection through the phase space can be written as

$$
\hat{L}=\frac{d}{d t}+\vec{v} \cdot \vec{\nabla}_{x}+\frac{\vec{F}}{m} \cdot \vec{\nabla}_{\nu}
$$

assuming the conjugate momentum has the simple form of velocity times mass. In General Relativity this has to be generalized to

$$
p^{\alpha} \frac{\partial}{\partial x^{\alpha}}-\Gamma_{\beta \psi}^{\alpha} p^{\beta} p^{\psi} \frac{\partial}{\partial p^{\alpha}}
$$

which simplifies in the FRW case to

$$
E \frac{\partial f}{\partial t}-\frac{\dot{R}}{R}|\vec{p}|^{2} \frac{\partial f}{\partial E}
$$

The total number density is obtained by integrating the distribution function over all momenta,

$$
n(t)=\frac{g}{(2 \pi)^{3}} \int d^{3} p f(E, t)
$$

Thus we obtain

$$
\frac{g}{(2 \pi)^{3}} \int d^{3} p \frac{\partial f}{\partial t}-\frac{\dot{R}}{R} \frac{g}{(2 \pi)^{3}} \int d^{3} p \frac{|\vec{p}|^{2}}{E} \frac{\partial f}{\partial E}=\frac{g}{(2 \pi)^{3}} \int C[f] \frac{d^{3} p}{E}
$$

Exchanging the order of integration and differentiation in the first term and working out the second term by doing an integration by parts, we can show that this equation becomes

$$
\frac{d}{d t} n+3 \frac{\dot{R}}{R} n=\frac{g}{(2 \pi)^{3}} \int C[f] \frac{d^{3} p}{E}
$$

Consider a process involving several particle species $\psi, a, b \ldots$

$$
\psi+a+b \leftrightarrow i+j+\ldots
$$

Our interest is usually a specific species which is undergoing an important change. We think of the collision operator with species $\psi$ as the object of main interest to be

$$
\begin{aligned}
\frac{g}{(2 \pi)^{3}} C[f] \frac{d^{3} p \psi}{d E \psi}= & -\int \frac{d^{3} p_{\psi}}{(2 \pi)^{3} 2 E_{\psi}} \times \frac{d^{3} p_{a}}{(2 \pi)^{3} 2 E_{a}} \times \ldots(2 \pi)^{4} \delta^{4}\left(p_{4}+p_{a} \ldots-p_{i}-p_{j} \ldots\right) \\
& \times\left[|M|_{\rightarrow}^{2} f_{\psi} f_{a}\left(1 \pm f_{a}\right)\right. \\
& \left.-|M|_{\leftarrow}^{2} f_{i}\left(1 \pm f_{a}\right)\left(1 \pm f_{\psi}\right)\right]
\end{aligned}
$$

where $\mathcal{M}$ represents a matrix element for the concerned process. There are Bose-Einstein and Fermi-Dirac distribution functions for the species in the in state. As for the out state, the \pm signs have to be chosen by knowing the species. Bosons prefer going into an occupied state (recall harmonic oscillation relation $a^{\dagger}|n\rangle=\sqrt{n+1}|n\rangle$ so that an $n$-tuply occupied state has weightage 
proportional to $n$ to be occupied). Hence the factors $(1+f)$, while fermions are forbidden from transiting to a state already occupied hence the Pauli suppression factors $(1-f)$. There is an integration over the phase space for each species.

Let us specialize the formalism further to cosmologically relevant case with isentropic expansion. Since the entropy density $s$ scales as $1 / R^{3}$, we can remove the presence of $R^{3}$ factors in number density of non-relativistic particles by studying the evolution of their ratio with $s$. Thus we define the relative abundance for a given species $Y=\frac{n \psi}{s}$. Then the we can see that

$$
\dot{n}_{\psi}+3 H n_{\psi}=s \dot{Y}
$$

Next the time evolution can be trade for temperature evolution in a radiation dominated universe, by introducing a variable $x$.

$$
x \equiv \frac{m}{T} \text { so that } \quad t=0.3 g_{*}^{-1 / 2} \frac{m_{P l}}{T^{2}}=0.3 g_{*}^{-1 / 2} \frac{m_{P l}}{m^{2}} x^{2} \equiv H^{-1}(m) x^{2}
$$

Thus we get the equation

$$
\begin{aligned}
\frac{d Y}{d x}= & -\frac{x}{H(m)} \int d \pi_{\psi} d \pi_{a}, \ldots d \pi_{i} \ldots(2 \pi)^{4}|M|^{2} \delta^{4}\left(p_{\text {in }}-p_{\text {out }}\right) \\
& {\left[f_{a} f_{b} \ldots f_{\psi}-f_{i} f_{j} \ldots\right] }
\end{aligned}
$$

Consider a species $\psi$ which is pair annihilating and going into a lighter species $X, \psi \bar{\psi} \rightarrow X \bar{X}$. The assumption is that the $X$ are strongly interacting either directly with each other or with rest of the contents, so that they equilibrate quickly and remain in equilibrium. Thus the species to be studies carefully is $\psi$. Due to the property of the chemical potential and detailed balance which would exist if the $\psi$ are also inequilibrium we can relate the equilibrium values

$$
n_{X} n_{\bar{X}}=n_{\psi}^{e Q} n_{\bar{\psi}}^{E Q}=\left(n_{\psi}^{E Q}\right)^{2}
$$

Note the superscript $e q$ is not necessary in the $n_{X}$ due to it always being in equilibrium. We can thus obtain the equation

$$
\frac{d Y}{d x}=-\frac{x s}{H(m)}\left\langle\sigma_{A}|\nu|\right\rangle\left(Y^{2}-Y_{E Q}^{2}\right)
$$

The solution of above equations can be simplified by identifying convenient regimes of values of $x$ in which approximate analytic forms of $Y_{E Q}$ exist

$$
\begin{aligned}
& Y_{E Q}(x)=\frac{45}{2 \pi^{4}}\left(\frac{\pi}{8}\right)^{1 / 2} \frac{g}{g_{* s}} x^{3 / 2} e^{-x} \quad x \gg 3 \text { non-relativistic case } \\
& Y_{E Q}(x)=\frac{45}{2 \pi^{4}} \xi(3) \frac{g_{e f f}}{g_{* s}}=0.278 \frac{g_{e f f}}{g_{* s}} \quad x \ll 3 \text { relativistic case }
\end{aligned}
$$

where the effective degeneracy factors $g_{\text {eff }}$ are defined relative to their usual values $g$ by $g_{\text {eff }}=$ $g_{\text {boson }}$ and $g_{\text {eff }}=\frac{3}{4} g_{\text {fermi }}$.

\subsubsection{Freeze out and subsequent evolution}

We can get further insight into the special case considered above, namely that of a species annihilating with its anti-particle and also going out of equilibrium. Define

$$
\Gamma_{A} \equiv n_{E Q}\left\langle\sigma_{A}|\nu|\right\rangle
$$


which represents the rate of the reactions, given as a product of the microscopic cross-section $\sigma$, and number density times relative velocity as a measure of the flux. Using this, we can rewrite the evolution equation above in the form

$$
\frac{x}{Y_{E Q}} \frac{d Y}{d x}=-\frac{\Gamma_{A}}{H}\left[\left(\frac{Y}{Y_{E Q}}\right)^{2}-1\right]
$$

This shows that the rate of approach to equilibrium depends on two factors. The second factor is the extent of departure from equilibrium, as we may expect even in a laboratory process. The front factor $\Gamma_{A} / H$ represents the competition between the annihilation rate (temperature dependent) and expansion rate ( also temperature dependent) of the Universe. When this front factor becomes small compared to unity, approach to equilibrium slows down, even if equilibrium is not reached. The abundance of the species $\psi$ in a comoving volume remains fixed once this factor becomes insignificant. This phenomenon is called "freeze out", i.e., the fact that the relative abundance does not change after this and continues to evolve like free gas.

After the species freezes out, at epoch $t_{D}$ with corresponding temperature $T_{D}$, the distribution function of the species continues to evolve purely due to the effect of the expanding spacetime. There are two simple rules of thumb we can prove for its distribution $d^{3} n / d^{3} p$ in phase space :

- A relativistic species continues to have the usual Bose-Einstein or Fermi-Dirac distribution function $\left(e^{\beta E} \pm 1\right)^{-1}$, except that $\beta^{-1}=T$ scales like $T(t)=T\left(t_{D}\right) R\left(t_{D}\right) / R(t)$.

- A species which is non-relativistic, i.e., mass $m \gg T_{D}$ the number density simply keeps depleting as $R^{-3}$, just like the particles which are still in equilibrium. But the momenta scale as $R^{-1}$, so energy $E=p^{2} / 2 m$ scales as $R^{-2}$. This is equivalent to the temperature scaling as $T(t)=T\left(t_{D}\right) R^{2}\left(t_{D}\right) / R^{2}(t)$.

Thus the distribution functions have an energy dependence which is simply obtained from their functional forms at the time of decoupling. In the relativistic case in fact remaining selfsimilar, and looks just like that of the particles still in equilibrium, with an important exception. If there is a change in the total number of effective degrees of freedom at some temperature, this information is not conveyed to the decoupled particles. In the non-relativistic case the scaling of the temperature parameter is significantly different.

\subsection{Dark Matter}

There is a variety of evidence to suggest that a large part of the matter content of the Universe is neither radiation, nor in the form of baryons. As such it is not capable of participating in processes producing electromagnetic radiation and christened Dark Matter.

The direct evidence for Dark Matter is available at two largely different scales. At the scale of individual galaxies and at the scale of clusters of galaxies. At the level of single galaxies it is possible to measure speeds of luminous bodies in the spiral arms for those galaxies which are visible edge on. The difference in the redshifts of the parts rotating away from us and the parts rotating towards us is measurable. It turns out that as a function of their distance from the center of the galaxy, velocities of rotation in the plane of the galaxy do not slow decrease in accordance with the $1 / r^{2}$ law expected from Kepler's law. Rather their speeds remain steadily high even beyond the visible edge of the galaxy. The plots of the velocity vs. the radial distance from the center of the galaxy have come to be called "rotation curves". The departure from Kepler law suggests presence of gravitating matter extended to ten times the size of the visible galaxy!

Secondly at the level of clusters of galaxies, it is possible to measure the relative speeds of the galaxies in a cluster, specifically the component of the velocity along the line of sight. By viirial theorem the values of these velocities should be set by the total matter content of the cluster. Again one finds the velocities more compatible with almost ten times the matter content compared to the visible. 
Another indicator of the extent of the baryonic content is indirect but very sensitive. Big Bang Nucleosynthesis predicts ratio of Hydrogen to Helium and the ratios of other light elements to Hydrogen determined by one parameter, the baryon to photon ratio, $\eta=B / s$ where $B$ is the net baryon number (difference of baryon and antibaryon numbers) and the denominator is the photon entropy. We shall have occasion to discuss this in greater detail in the section on Baryogenesis. The observed ratios of Helium to Hydrogen and other light nuclei to Hydrogen is correctly fitted only if $\eta \sim 10^{-9}$. Knowing the photon temperature very accurately we know the contribution of radiation to the general expansion (it is very insignificant at present epoch). Further knowing this accurately we know the baryon abundance rather accurately. Between the two, the latter is certainly the dominant contribution to the energy density of the present Universe. However the total amount of matter-energy required to explain the current Hubble expansion is almost 30 times more than the abundance baryons inferred through the BBN data. Again we are led to assume the existence of other forms of matter energy that account for the Hubble expansion. It is therefore assumed that there is extensive amount of non-relativistic matter present in the Universe today, and is called Dark Matter. We do not know at present whether Dark Matter is a single species of particles or several different species of particles. We do not know the possible values for the masses of such particles, however the study of galaxy formation suggests two classes of Dark Matter distinguished by their mass as we see in the next paragraphs.

The latest data from all sources suggest that the dominant component of the energy driving the expansion is actually neither radiation nor matter, but some other form of energy obeying an equation of state close to that of relativistic vacuum, $p=-\rho$. This is estimated to be contribute about $70 \%$. The Dark matter component is estimated to be about $27 \%$, and only about $3 \%$ in the form of baryonic matter. These conclusions follow from WMAP data on CMB and type Ia supernova data on expansion rate of the Universe more than 7 billion years ago. It is remarkable that the approximately 10 times the abundance of Dark Matter relative to baryonic matter as inferred directly from galacic and cluster data is verified reasonably accurately by the very indirect methods. This is what gives us confidence in the Dark Matter hypothesis.

When galaxy formation is considered this highly abundant Dark Matter component plays a significant role. While no other kind of interaction is permitted between baryonic matter and Dark Matter at least at low energies, gravity is always a mediator. It is no surprise therefore that the Dark Matter is clustered in approximately the same way as luminous baryonic matter. The question whether there are large distributions of Dark Matter separately from baryonic matter needs experimentally studied however so far the evidence does not seem to demand such an assumption.

It then follows that the growth of perturbations which led to galaxy formation must have proceeded simultaneously for the baryonic matter and the Dark Matter, coupled to each other through gravity. The study of this coupled evolution gives rise a distinction of two categories of Dark Matter which can be made based on the mass of the corresponding particle. Those particles that have become non-relativistic by the time of galaxy formation are called Cold Dark Matter ( CDM). They are in the form of pressureless dust by this epoch and their chief contribution to energy density comes from their rest masses and not their thermal motion, hence Cold. We may think of this dividing line as set by the temperature $\sim 1 \mathrm{eV}$ when neutral Hydrogen forms. Particles which are already non-relativistic at this temperature certainly belong to the category of CDM. On the other hand particles that remain a relativistic gas down to $1 \mathrm{eV}$ temperature contribute through their thermal energy density and are called Hot Dark Matter ( HDM). A prime candidate for this kind of DM is a neutrino, whose masses are constrained to very small values.

The main difference in the two kinds of DM comes from the nature of the clustering they assist. From Jeans formula we see that HDM clustering occurs at large physical scales while CDM can cluster at much smaller scales. In fact too much HDM can destroy clumping of baryonic clusters at smaller scales. Thus a study of the spectrum of perturbations $\mathcal{P}(k)$ gives a clue to the form of DM that assisted the formation of galaxies. The current evidence in the light of the WMAP data strongly suggests essentially the presence only of CDM, though some proportion of a HDM species cannot be ruled out.

In the following subsections we shall show how we can trace back at least some of the micro- 
scopic properties of the Dark Matter if we know its abundance today.

\subsubsection{Hot Relics}

For particles that continue to remain relativistic as they are going out of equilibrium, the equations from the previous subsection can can be used to show that their abundance at late time is determined by the value of their freeze out temperature, i.e., $x_{\text {freeze out }}$

$$
Y_{\infty}=Y_{E Q}\left(x_{\text {freeze out }}\right)=0.278 \frac{g_{\text {eff }}}{g_{* s}(x)}
$$

If we want to think of this as the Dark Matter candidate, we estimate the energy density it can contribute, which is determined to be

$$
\rho_{\psi_{o}}=s_{0} Y_{\infty} m=3 Y_{\infty}\left(\frac{m}{e V}\right) \mathrm{keV}-(\mathrm{cm})^{-3}
$$

From LSS data on distribution of fluctuations, as also the WMAP data it is now concluded that the structure formation could not have occurred due to HDM. Hence this is not a very useful quantity to verify against observations. Historically, this density value was used to put an upper bound on the mass of a neutrino. If the decoupled neutrino is to not be so overabundant that it exceeds the current density of the Universe, than its mass must be bounded.

$$
m \lesssim 13 e V \frac{g_{* s}\left(x_{f}\right)}{g_{\text {eff }}}
$$

For $\nu$ 's the ratio of the $g_{*}$ factors is 0.14 , from which one can conclude that $m_{\nu}<91 \mathrm{eV}$. This is known as the Cowsik-McClelland bound. Although the bound is surpassed by both by terrestrial experiments and recent astrophysical data, it is an instructive exercise.

\subsubsection{Cold Relics}

For cold relics, we need to determine the quantities $x_{f}, T_{f}$ corresponding to the freeze out of the species, and its present abundance relative to radiation, $Y_{\infty}$. These are determined by solving the equation

$$
\frac{d Y}{d x}=-\frac{1}{x^{2}} \sqrt{\frac{\pi g_{*}(T)}{45}} M_{P}\langle\sigma v\rangle\left(Y(T)^{2}-Y_{e q}(T)^{2}\right)
$$

It is useful to make an expansion of the cross-section in partial waves, which amounts to an expansion in energy, or equivalently in the present setting, an expansion in $x=m / T$. For a massive particle the leading term is

$$
\left\langle\sigma_{a}|v|\right\rangle \equiv \sigma_{o}\left(\frac{T}{m}\right)^{n}=\sigma_{o} x^{-n} \quad x \gtrsim 3
$$

Thus expressing the cross-section as a function of $x$, the equation can be solved. The solution to this equation gives the left over abundance for a massive particle $\chi$ at present time. The answer typically has the following dependence

$$
Y_{\infty}=O(1) \times \frac{x_{f}}{m_{\chi} M_{P}\left\langle\sigma_{A}|v|\right\rangle}
$$

with $x_{f}$ determined numerically when the $Y$ effectively stops evolving. The present contribution to the energy density due to these particles is $m_{\chi} Y_{\infty} \times\left(s\left(T_{0}\right) / \rho_{\text {crit }}\right)$ where $s\left(T_{0}\right)$ is the present value of entropy density in radiation.

It is thus possible to relate laboratory properties of the $\chi$ particle with a cosmological observable. Given a particle physics model, we can constrain the properties of the potential Dark Matter candidate by calculating its contribution to $\Omega_{D M}$ and then counterchecking the same cross-section in collider data. 


\section{Missing Dark Energy}

The important topic of Dark Energy could not be included within the limitations of this course. The reader can refer to some of the excellent reviews cited at the end or await the next avatara of these notes.

\section{Baryon asymmetry of the Universe}

A very interesting interface of Particle Physics with cosmology is provided by the abundance of baryons in the Universe. At first it is a puzzle to note that we only have baryonic matter present in the Universe, with no naturally occurring baryons to be seen.

In principle a cluster of galaxies completely isolated from others could be made totally from anti-Hydrogen and anti-elements. However there should be some boundary within which this confined, since any contact with usual baryonic matter would generate violent gamma ray production which would be observed as a part of cosmic rays. But there are no clearly visible empty corridors separating some galaxies or clusters of galaxies from others, nor is there a significant gamma ray background to indicate ongoing baron-anti-baryon annihilation. Thus we assume the present Universe to be devoid of priordial anti-baryons.

Due to charge neutrality, the electron number should be exactly equal to the proton number of the Universe, and if Lepton number were conserved, we should therefore have a predictable abundance of electron type anti-neutrinos. However after the discovery of neutrino oscillations the question of total lepton number conservation is open and their number may not be determined exactly by the charged lepton number. Thus the total matter vs. anti-matter asymmetry of the Universe is a more complicated question. We shall deal only with the baryons where the situation is more precisely known.

The observed asymmetry is quantified by expressing it as a ratio of the photon number density, i.e., entropy,

$$
\eta \equiv \frac{n_{B}}{s} \equiv \frac{n_{b}-n_{\bar{b}}}{n_{\gamma}}
$$

where the upper case subscript $B$ signifies the net baryon number while the lower case subscripts $b, \bar{b}$ signify the actual densities of baryonic and anti-baryonic species separately. Big Bang nucleosynthesis constrains the value of this ratio very precisely. The abundances of Helium ${ }^{4} \mathrm{He}$ to Hydrogen is sensitively dependent on this ratio, but further, the abundances of light nuclei such as Deuterium, ${ }^{3} \mathrm{He}$, and ${ }^{7} \mathrm{Li}$ relative to Hydrogen are also extremely sensitive to this ratio.

\subsection{Genesis of the idea of Baryogenesis}

We believe that the Universe started with a hot Big Bang. If the laws of nature were completely symmetric with respect to matter and anti-matter both should be present in exactly same abundance in thermodynamic equilibrium. Then the asymmetry between the two has to be taken as an accidental initial condition. Fortunately we know that the Weak interactions do not respect charge conjugation or matter-anti-matter symmetry $C$, but only the product $C P$ after parity $P$ is included. Further, in 1964-65 two crucial discoveries were made. It was shown that certain sectors of the theory $\left(K^{0}-\bar{K}^{0}\right.$ system) also do not respect $C P$. In QFT there is a theorem that says that it is impossible to write a Lorentz invariant local theory which does not respect the combination $C P T$, now including time reversal $T$. Thus a $C P$ violating theory presumably violates $T$ invariance in same measure. The small mixing of $C P$ eigenstates will also be reflected in small asymmetry in reaction rates involving these participants.

The other crucial discovery was the Cosmic Microwave Background which established the Hubble expansion back to temperatures as high as $1000 \mathrm{~K}$. It is easy to extrapolate this to sufficiently early times and higher temperatures when density and temperature would be sufficiently high for Particle Physics processes to occur freely. The stage was set for searching for a dynamical expla- 
nation for the baryon asymmetry (Weinberg 1964) and a specific model was proposed (Sakharov 1967).

\subsection{Sakharov criteria}

The minimal conditions required for obtaining baryon asymmetry have come to be called Sakharov criteria. They can be understood via a specific example. Consider a species $X$ which carries baryon number and is decaying into two different possible final products, either two quarks or one antiquark and a lepton. (We use one of the decay modes to determine the baryon number of $X$ and violation shows up in the other decay). Such decays are easily possible in Grand Unified models. The following should be true for a net $B$ number to remain in the Universe :

1. Baryon number violation

$$
X \rightarrow \begin{array}{cc}
q q & \Delta B_{1}=2 / 3 \\
\bar{q} \bar{\ell} & \Delta B_{2}=-1 / 3
\end{array}
$$

2. Charge conjugation violation

$$
\mathcal{M}(X \rightarrow q q) \neq \mathcal{M}(\bar{X} \rightarrow \bar{q} \bar{q})
$$

3. $\mathrm{CP}$ violation reflected in difference in rates

$$
r_{1}=\frac{\Gamma_{1}(X \rightarrow q q)}{\Gamma_{1}+\Gamma_{2}} \neq \frac{\bar{\Gamma}_{1}(\bar{X} \rightarrow \bar{q} \bar{q})}{\bar{\Gamma}_{1}+\bar{\Gamma}_{2}}=\bar{r}_{1}
$$

4. Out-of-equilibrium conditions, which would make reverse reactions become unfavorable

$$
\begin{aligned}
\text { Net } B= & \Delta B_{1} r_{1}+\Delta B_{2}\left(1-r_{1}\right) \\
& \left(-\Delta B_{1}\right) \bar{r}_{1}+\left(-\Delta B_{2}\right)\left(1-\bar{r}_{1}\right) \\
= & \left(\Delta B_{1}-\Delta B_{2}\right)\left(r_{1}-\bar{r}_{1}\right)
\end{aligned}
$$

In the early Universe, the condition for departure from Equilibrium means that the reaction rate should become slow enough to be slower than the Hubble expansion rate at that epoch. This will happen because reaction cross-sections depend both on density which is falling due to expansion, and the energy dependence of the intrinsic cross-section makes it smaller at lower temperature.

$$
\begin{aligned}
\Gamma_{X} & \simeq \alpha_{X} m_{X}^{2} / T \\
H & \simeq g_{*}^{1 / 2} T^{2} / M_{P l}
\end{aligned}
$$

Need the rate $\Gamma_{X}$ still $<H$ when $k T \sim m_{X}$. Thus $k T_{D} \sim\left(\alpha_{X} m_{P L} m_{X}\right)^{1 / 2}$.

Resulting

$$
\frac{n_{B}}{s} \simeq \frac{B}{g_{*}} \times(\text { Boltzmann evolution })
$$

Thus the result depends purely on the microscopic quantity $B$ (includes $\delta_{C P}$ ) and $g_{*}$ of the epoch when the mechanism operates. 


\subsection{Anomalous violation of $B+L$ number}

Quantization of interacting field theories contain many subtleties. Sometimes the process of renormalization does not respect a symmetry present at the classical level. Then quantum mechanically the corresponding number operator is not conserved. This situation is called anomaly. This is tolerable if it happens for a global charge. If it happens for a gauge charge the model would not be viable. It turns out that the Standard Model of Particle Physics does not respect the global quantum number $B+L$, baryon number plus lepton number. The number $B-L$ is also not coupled to any gauge charge howeve it remains, miraculously, anomaly free and hence is conserved.

The anomalous violation is not obtained in perturbation theory. However a handle on the anomalous violation rate can be obtained by topological arguments involving global configurations of gauge and Higgs fields. A specific configuration whose energy value determines the rate is called sphaleron. The energy of a sphaleron is approximately $5 \mathrm{TeV}$ in Standard Model. At temperatures well below this, the rate is suppressed exponentially. At a temperatrue much higher, the rate is order unity. Actually it becomes meaningless to speak of a conserved number. However a number generated by any other mechanism will be quickly equilibrated to zero by this non-conservation.

In the in between regime of temperatures, the rate is estimated as

$$
\Gamma \approx \kappa(\mathcal{N} \mathcal{V})_{0} T^{4} e^{-E_{s p h}(T) / k T}
$$

where $\kappa$ is the determinant of other fluctuations (recall Coleman tunneling formula) and $\mathcal{N} \mathcal{V}_{0}$ represents sphaleron zero-mode volume, i.e., the weightage associated with all the possible ways a sphaleron can arise. This formula is valid for $m_{W} \ll T \ll m_{W} / \alpha_{W}$ where $m_{W}$ is mass of the $W$ boson and $\alpha_{W}$ is the fine structure constant $g^{2} / 4 \pi$ of the Weak interactions.

Sphaleron energy depends on the Higgs mass at zero temperature in such a way that too light a Higgs $(<90 \mathrm{GeV})$ would result in very rapid violation of $B+L$ around the electroweak phase transition. The conclusion is that either the Higgs is heavier ( which is corroborated by the bound $m_{H}>117 \mathrm{GeV}$ from LEP data), or there is more than one Higgs, or that there was a primordial $B-L$ number already present at the electroweak scale.

\subsection{Electroweak baryogenesis}

Could the baryon number arise at the elctroweak scale itself? Sphaleronic processes are already becoming unimortant at this scale. Also the properties any new particles needed can be counterchecked at the LHC or ILC. At the electroweak scale the expansion of the Universe is many orders of magnitude $\left(10^{12}\right)$ slower than the particle physics processes. Hence direct competition with rates is not possible. However, a first order phase transition leads to formation of bubble walls. They sweep past any given point in sufficiently short time scale that Particle Physics scales compete with this time scale rather than the expansion time scale of the Universe. Such a scenario which by-passes the thermal conditions in the Universe is called non-thermal, as against the example studied at the beginning of the section which is called thermal mechanism for baryogenesis.

If we enhance the SM with additional particles we can actually use the sphaleronic excitations to generate $B+L$ asymmetry if the other criteria of Sakharov are satisfied. Typical scenarios rely on

1. Usual $C$ asymmetry of Weak interactions

2. $B+L$ violation by sphaleronic excitations

3. $C P$ violation due to complex phases in the vacuum expectation values of one or more scalar fields

4. Out-of-equilibrium conditions due a first order phase transition

It turns out that all of these conditions are easily satisfied provided we have more than one Higgs scalar and sufficiently large $C P$ phases entering some fermion masses. In specific models favored for esthetic reasons however it has not been easy to reconcile all the known constraints from other 
data with the requirements of electroweal baryogenesis. For example, the Minimal Supersymmetric Standard Model (MSSM) has the following dangers ( see M. Quiros hep-ph/0101230)

- Need for first order phase transition implies a light Higgs and a light superpartner "stop" of the top quark, as also a bound on the ratio of the masses of the two neutral Higgs bosons expressed as $\tan \beta$,

$$
110<m_{H}<115 \mathrm{GeV}, \tan \beta \lesssim 4, m_{\tilde{t}_{R}} \sim 105 \operatorname{to} 165 \mathrm{GeV}
$$

- One requires $\delta_{C P} \gtrsim 0.04$ which in turn raises the danger of color breaking vacua.

\subsection{Baryogenesis from Leptogenesis}

A realistic alternative possibility for dynamical explanation for baryon asymmetry is thrown up by the discovery of neutrino mass. The very small mass $m_{\nu} \sim 0.01 \mathrm{eV}$ for neutrinos requires their Yukawa coupling to the Higgs to be $10^{-11}$. As we discussed in case of inflation, such small dimensionless numbers seem to hide some unknown dynamics going on. A very elegant explanation for the small mass can be given if we assume (i) Majorana type masses for the neutrinos and (ii) assume this mass, denoted $M_{R}$ to be high, $M_{R} \sim 10^{14} \mathrm{GeV}$. It can be shown that

$$
m_{\nu} M_{R} \simeq m_{W}^{2}
$$

is a natural relation if such a scale is assumed. Now a scale like $10^{14}$ is also far removed from other physics, but is tantalisingly in the range of Grand Unified theories. This mechanism is called see-saw mechanism

This possibility makes leptogenesis naturally possible in the early Universe almost literally by the example we studied earlier for the particle $X$ at the beginning of the section. Majorana fermions do not conserve fermion number. Further, the mixing of the three generations can introduce a complex phase in the mass matrix which can lead to $C P$ violation. Finally high mass means that the decay rate can actually compete with the expansion scale of the Universe which is sufficiently rapid at high temperatures, unlike at electroweak scale. This can result in lepton asymmetry of the Universe. This lepton asymmetry converts to baryon asymmetry as follows. Recall that at temperatures high compared to the electroweak scale, $B+L$ number is meaningless, and will be equilibrated to zero. That is, the anomalous effects ensure $\Delta(B+L)=0$ and hence will generate approximately $\Delta B \sim-\Delta L$. The equality is not exact due to interplay of several chemical potentials one has to keep track of.

An important handle on this proposal is provided by the low energy neutrino data. It is possible to constrain the extend of $C P$ violation that can be available at high scale from low scale masses due to see-saw mechanism. Consider the decay of a heavy neutrino species $N$ into a light lepton $\ell$ and a Higgs particle. There are several such possibilities, and in each case the electric charge in the final state is balanced to be zero. Due to lepton number violation characteristic of Majorana fermions, the same $N$ can also decay into anti-lepton and anti-Higgs. Thus the difference in the lepton number of the final products in the two different modes is $\Delta L=2$ along the same lines as $\Delta B=1$ in our example at the beginning of the section. Then the $C P$-asymmetry parameter in the decay of any one of the three heavy neutrinos $N_{i}, i=1,2,3$ is defined as

$$
\epsilon_{i} \equiv \frac{\Gamma\left(N_{i} \rightarrow \bar{\ell} \phi\right)-\Gamma\left(N_{i} \rightarrow \ell \phi^{\dagger}\right)}{\Gamma\left(N_{i} \rightarrow \bar{\ell} \phi\right)+\Gamma\left(N_{i} \rightarrow \ell \phi^{\dagger}\right)} .
$$

If we assume a hierarchy of masses $M_{1}<M_{2}<M_{3}$ as is the case of all other fermions, then the main contribution to the lepton asymmetry generation comes from the species to decay last, i.e., the lightest of the heavy neutrinos $N_{1}$. (Why?) The maximum value of CP violation parameter $\epsilon_{1}$ in this case can be shown to be

$$
\left|\epsilon_{1}\right| \leq 9.86 \times 10^{-8}\left(\frac{M_{1}}{10^{9} \mathrm{GeV}}\right)\left(\frac{m_{3}}{0.05 \mathrm{eV}}\right) .
$$


where the mass of the heaviest of the light neutrinos $\nu_{3}$ is bounded by the atmospheric neutrino data, which gives the mass-squared difference $\Delta m_{a t m}^{2} \equiv m_{3}^{2}-m_{1}^{2}$. Thus, $m_{3} \simeq \sqrt{\Delta m_{a t m}^{2}}=0.05 \mathrm{eV}$.

In the figure 8 we show the solutions of the Boltzmann equations showing the accumulation of $B-L$ as temperature $T$ drops for various values of $M_{1}$ with $C P$ violation chosen to be maximal permissible according to above formula and the parameter $\widetilde{m_{1}}=\left(m_{D}^{\dagger} m_{D}\right)_{11} / M_{1}$ chosen $10^{-5} \mathrm{eV}$. It turns out that this particular parameter ( numerator is the 11 element of the square of Dirac mass matrix for the neutrinos) determines the overall outcome of the thermal $B-L$ number production. We see that there is negligible net number $B-L$ at high temperature but it builds

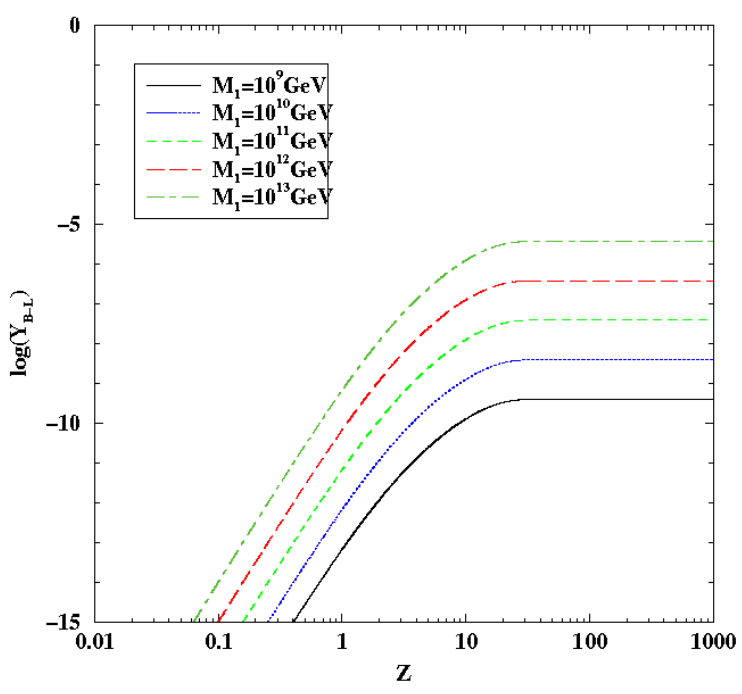

Figure 8: The evolution of the $B-L$ asymmetry with temperature, shown here as a function of $Z=M_{1} / T$, with fixed values of $M_{1}$ as indicated in the legend. The value of the $C P$ violation parameter is maximal permissible and the parameter $\widetilde{m_{1}}$ explained in text is chosen $10^{-5} \mathrm{eV}$ for all graphs. Figure from N. Sahu et al, Nucl. Phy. B752 (2006)

up as the decay processes are going out of equilibrium. At some point the production levels off. Then due to sphalerons, the asymmetry which is initially in the form of light neutrinos also gets converted to baryonic form producing net $B$ number.

From such exercises it can be shown that we need the mass scale $M_{R}$ of the heavy majorana neutrinos to be typically $>10^{12} \mathrm{GeV}$ but with some optimism, at least $>10^{9} \mathrm{GeV}$ for successful thermal leptogenesis. The problem with this conclusion is that firstly a new intermediate scale much lower than required for gauge coupling unification is called for. Secondly, as discussed in the Introduction, we expect supersymmetry to regulate the QFT involved in Grand Unification with several scales of symmetry breaking. But supersymmetry necessarily implies the existence of gravitino. Further, it can be shown that if our Universe underwent simple radiation dominated expansion from any temperature larger than $10^{9} \mathrm{GeV}$ down to Big Bang Nucleosynthesis, sufficient number of gravitinos would be generated that would make the Universe matter dominated and foul up BBN. Thus it is usual to assume that the "reheat" temperature after inflation is lower than $10^{9} \mathrm{GeV}$. But then the thermal leptogenesis discussed here becomes unviable.

It remains an open possibility that there are non-thermal mechanisms similar to the electroweak baryogenesis, but applicable to leptogenesis. 


\section{Appendix}

Here we discuss the "True or False" statements given in section 1.1] Note that some of the statements are half baked and warrant a discussion rather than simple yes or no. Some hints.

1. Curved spacetime takes account of equivalence of gravitational and innertial mass. The Relativity principle of space and time could have been Galilean and the formulation would be still useful. See ref [1], chapter 12 for Cartan's formulation.

2. Reparametererization only relabels points. It cannot change physics. Usually the laws are written only in the form invariant under rigid rotations. But every law can in principle be rewritten to be form invariant under change of parameterization. Thus reparameterization invariance cannot be a new physical principle.

3. Due to Equivalence Principle as adopted by Einstein, all forms of energy are subject to and contribute to gravitational field. Energy density therefore must contain contribution of gravitational "binding energy". However we can always choose freely falling frames locally so that effect of gravity disappear. In these frames the energy density of gravitational field disappears.

4. Total energy would be an integral of the energy density over a whole spacelike surface. This answer would remain unchanged under coordinate local transformations especially if we restrict ourselves to rigid transformations at infinity ( sitting where we measure up the energy). But GR throws up the possibility of compact spacelike hypersurfaces. In this case asymptotic region is not available.

5. If this genuinely means spacetime measurements are meaningless at that point then it is unphysical. But it can be an artifact of coordinate system, as for instance the origin in a spherical or cylindrical coordinates.

6. Divergence of metric coefficients is often avoided using different coordinate systems.

7. Curvature tensor is a physical quantity. Divergence of its components will also often imply divergence of some components of energy-momentum tensor. Such points would be unphysical. However note that much electrostatics is done assuming point charges. These have infinite energy density at the location of the point. When such points are isolated we hope some other physics takes over as the singular point is approached.

8. The expansion of the Universe is neither relativistic, nor a strong gravity phenomenon at least ever since BBN. It admits Newtonian description. If the spacelike hypersurfaces were compact that would be easier to explain as a dynamical fact in GR. In Newtonian physics we would simply accept is as fact, just as we are willing to accept infinite space as fact. 


\section{References}

1. Historical perspective along with personalities and an emphasis on a modern geometric view of General Relativity can be found through out the textbook by C. W. Misner, K. S. Thorne and J. A. Wheeler, "Gravitation" W. A. Freeman Co. (1973).

2. Emphasis on aspects of General Relativity which keep it on par with other field theories of High Energy Physics, is presented in S. Weinberg, "Gravitation and Cosmology" John Wiley and Sons (1973)

3. E. W. Kolb and M. S. Turner, "The Early universe", Addison-Wesley Pub. Co. (1990)

4. T. Padmanabhan "Theoretical Astrophysics, vol III : Galaxies and Cosmology" Cambridge University Press (2002)

5. G. Lazarides, "Basics of inflationary cosmology", Corfu Summer Institute on Cosmology, J. Phys. Conf. Ser. 53, 528 (2006) arXiv:hep-ph/0607032.

6. A. Riotto "Inflation and the Theory of Cosmological Perturbations", Summer School on Astroparticle Physics and Cosmology, Trieste, (2002) arXiv:hep-ph/0210162

7. R. H. Brandenberger "Lectures on the Theory of Cosmological Perturbations" Lectures at summer school in Mexico, arXiv:hep-th/0306071

8. T. Padmanabhan "Understanding our Universe : current status and open issues" in 100 Years of Reality - Space-time Structure: Einstein and Beyond, A. Ashtekar Ed., World Scientific Pub. Co., Singapore (2005), arXiv:gr-qc/0503107 
This figure "StdVsInfCosmo.png" is available in "png" format from: http://arxiv.org/ps/0808.2236v1 
This figure "Vbarrier.png" is available in "png" format from: http://arxiv.org/ps/0808.2236v1 
This figure "VeffT.png" is available in "png" format from: http://arxiv.org/ps/0808.2236v1 
This figure "bounce.png" is available in "png" format from: http://arxiv.org/ps/0808.2236v1 
This figure "highf.png" is available in "png" format from: http://arxiv.org/ps/0808.2236v1 
This figure "lowf.png" is available in "png" format from: http://arxiv.org/ps/0808.2236v1 
This figure "maxwell-graph.png" is available in "png" format from: http://arxiv.org/ps/0808.2236v1 
This figure "photodec.png" is available in "png" format from: http://arxiv.org/ps/0808.2236v1 
This figure "thasy13-5.png" is available in "png" format from: http://arxiv.org/ps/0808.2236v1 